\title{
DPP-4 enzyme deficiency protects kidney from acute ischemia- reperfusion injury: role for remote intermittent bowel ischemia- reperfusion preconditioning
}

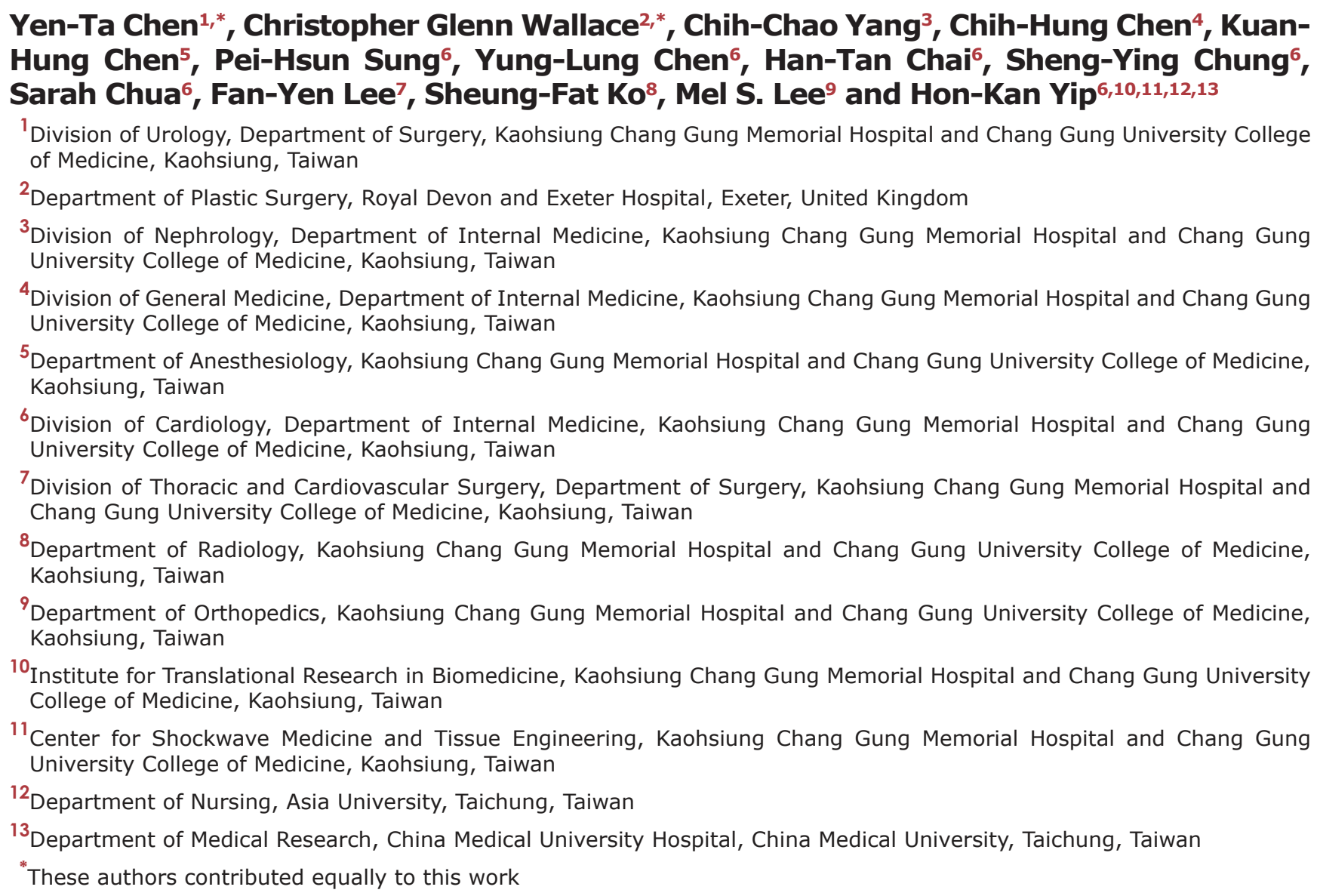

Correspondence to: Hon-Kan Yip, email: han.gung@msa.hinet.net

Keywords: acute kidney ischemia-reperfusion injury, preconditioning, inflammation, oxidative stress, dipeptidyl peptidase 4 deficiency

Received: January 27, $2017 \quad$ Accepted: June 17, $2017 \quad$ Published: July 04, 2017

Copyright: Chen et al. This is an open-access article distributed under the terms of the Creative Commons Attribution License 3.0 (CC BY 3.0), which permits unrestricted use, distribution, and reproduction in any medium, provided the original author and source are credited.

\section{ABSTRACT}

We analyzed the effects of acute ischemia-reperfusion (KIR) injury on the status of kidney function and architecture in dipeptidyl peptidase4-difficient (DPP4 ${ }^{\mathrm{D}}$ ) rats and the effect of remote small bowel ischemia-reperfusion (BIR) preconditioning. DPP4deficient (DPP4 ${ }^{\mathrm{D}}$ ) and normal Fischer344 (F344) rats were divided into 6 groups: (1) sham-F344, (2) sham-DPP4', (3) KIR-F344 (4) KIR-DPP4', (5) DPP4' ${ }^{\mathrm{D}}$ KIRextendin-9-39 and (6) BIR-KIR-F344. Blood creatinine and urea nitrogen levels and the urinary protein-to-creatinine ratio was higher in KIR-F344 rats than BIR-KIR-F344 or KIR-DPP4 ${ }^{\mathrm{D}}$ rats $\mathbf{7 2} \mathrm{h}$ after acute KIR. Conversely, the circulating glucagon-like peptide 1 (GLP-1) levels were higher in BIR-KIR-F344 and KIR-DPP4' ${ }^{D}$ than KIR-F344 rats after acute KIR. KIR-F344 rats showed greater inflammation, oxidative stress, 


\begin{abstract}
apoptosis, DNA damage and kidney injury than other rat groups. Damage to the kidney architecture in KIR-F344 rats was greater than in BIR-KIR-F344 or KIR-DPP4D rats. Expression of antioxidant proteins and GLP-1 receptor was higher in kidneys from KIRDPP4' and BIR-KIR-F344 than KIR-F344 rats, which suggests better intrinsic responses. We therefore suggest that elevated circulating GLP-1 levels due to DPP4 deficiency and BIR preconditioning protect kidney function and architecture during acute IR injury.
\end{abstract}

\section{INTRODUCTION}

Despite novel drugs and improvements in critical care including renal replacement therapy, the outcomes for critically ill acute kidney injury (AKI) patients remains poor and its incidence during hospitalization keeps increasing [1-6]. Acute kidney ischemia-reperfusion (KIR) that causes AKI is frequently observed during contrast-media induced nephropathy [5], post-resuscitation shock [7], kidney transplantation [8] and chemical or drug toxicity. These result in acute tubular-epithelial damage $[9,10]$, loss of tubular microvasculature [11] and inflammation and leukocyte infiltration [8-10, 12].

Acute KIR injury involves oxidative stress and reactive oxygen species (ROS) [13-15], mitochondrial damage [13-15], apoptosis [11,13-15], and complement cascade and inflammation [11-15]. Therefore, suppression of inflammation and oxidative stress/ROS may be beneficial for AKI patients.

Glucagon-like peptide-1 (GLP-1) based drugs are emerging therapeutics for type 2 diabetes mellitus (DM). GLP-1 reduces inflammation and oxidative stress/ ROS [14, 16-18] and the GLP-1 receptor (GLP-1R) is expressed in the brain, kidney, digestive organs and heart and upregulated during IR injury [14, 19-21]. Therefore, increased circulating levels of GLP-1 may protect the kidney from acute IR injury.

GLP-1 is the substrate of dipeptidyl peptidase 4 (DPP4) or CD26, which is a membrane-anchored ecto-protease that cleaves GLP-1. Hence, pharmacological inhibition of DPP4 enhances circulating GLP-1 levels [21-25] in animal models of acute kidney IR injury [14, 21, 23]. However, the direct association between DPP4 and GLP-1 has not been demonstrated, especially in regard to protection from acute IR injury [14, 15, 21, 24, 25].

The role of ischemic preconditioning in protecting organs from ischemic injury has been the focus of many investigations, especially related to the acute coronary syndrome [26-28]. Studies have shown that remote organ ischemia-related preconditioning protects other organs/ tissues against IR injury [29-31]. Especially, intestinal ischemic preconditioning suppresses oxidative stress and protects remote organs from ischemic reperfusion damage $[32,33]$. These findings suggest that intermittent small bowel IR (BIR) may protect kidneys from acute IR injury since the gastro-intestinal tract secretes GLP-1 [26-33]. Therefore, in this study, we tested if DPP4 deficiency and intermittent BIR preconditioning protected kidneys against acute IR injury.

\section{RESULTS}

\section{Circulating creatinine and blood urea nitrogen (BUN) levels and urine protein to creatinine ratio at baseline and day 3 after acute KIR}

Before the IR procedure, blood urea nitrogen (BUN) and blood creatinine were similar in all 6 groups of rats. Similarly, the protein to creatinine ratios in the urine was similar in the 6 groups of rats (Figure 1). However, $72 \mathrm{~h}$ after acute KIR, blood creatinine and BUN levels that indicate renal dysfunction, and the urine protein to creatinine ratio that indicate glomerular damage were lowest in the SC-F344 and SC-DPP4 ${ }^{\mathrm{D}}$ rats and gradually increased in BIR-KIR-F344, KIR-DPP4 ${ }^{\mathrm{D}}$, DPP4D-KIRexendin-9-39 rats and were highest in KIR-F344 rats (Figure 1).

Kidney injury score and circulating GLP-1 levels on day 3 after acute KIR

We scored the H\&E stained sections for kidney injury and found that SC-F344 and SC-DPP4 $4^{\mathrm{D}}$ rats had lowest scores. The scores were gradually higher for BIRKIR-F344, KIR-DPP4 ${ }^{\text {D, DPP4 }}{ }^{\text {D-KIR-exendin-(9-39) rats }}$ and were highest in KIR-F344 rats (Figure 2).

The baseline circulating levels of GLP-1 were higher in the SC-DPP4 ${ }^{\mathrm{D}}$ rats than SC-F344 rats. Further, the circulating levels of GLP-1 on day 3 after acute KIR procedure were significantly lower in the SC-F344 than KIR-F344 and KIR-BIR-F344 group rats, which had similar GLP-1 levels. Circulating GLP-1 levels were higher in the KIR-DPP4 $4^{\mathrm{D}}$ group and highest in KIRDPP4 ${ }^{\text {D }}+$ Ex4-9-39 group compared to the SC-DPP4 ${ }^{\text {D }}$ group. The higher circulating GLP-1 levels on day 3 after acute KIR in the KIR-DPP4 + Ex4-9-39 rats compared to KIR-BIR-F344 rats suggested enhanced intrinsic response to the occupied GLP-1R in kidney parenchyma elicited by Ex4-9-39 (Figure 2).

Time course analysis of circulating GLP-1 levels in BIR-KIR-344 and BIR-344 animals showed that they were markedly upregulated only at 3-6 h after BIR-KIR procedure suggesting that GLP-1 elevation was in response to IR (Figure 2). 
A

$\mathbf{O} \mathbf{h}$

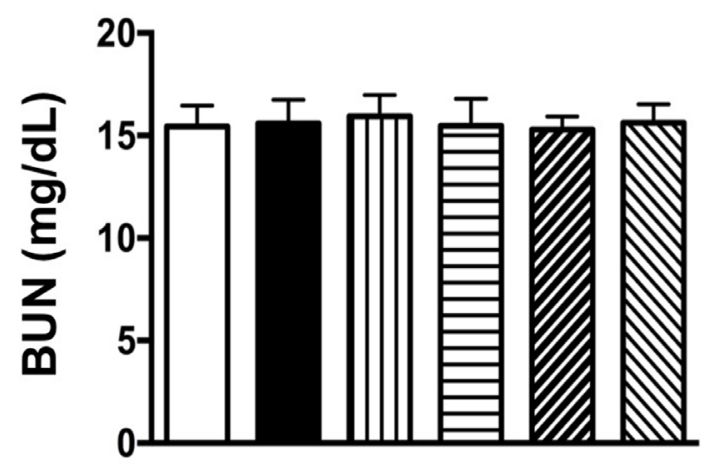

C

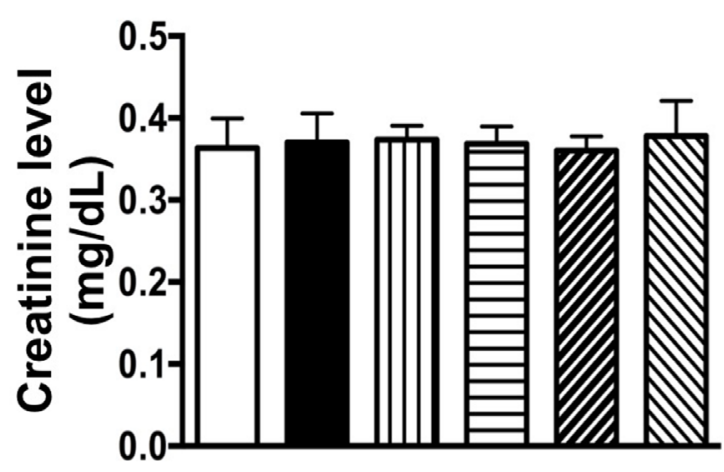

E

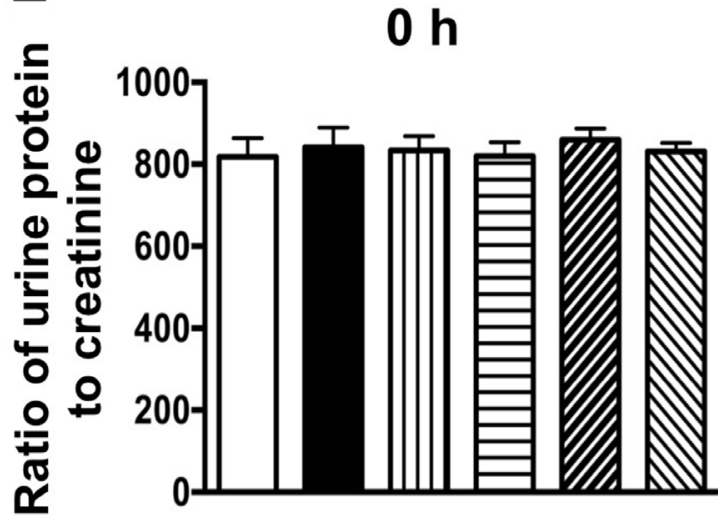

B

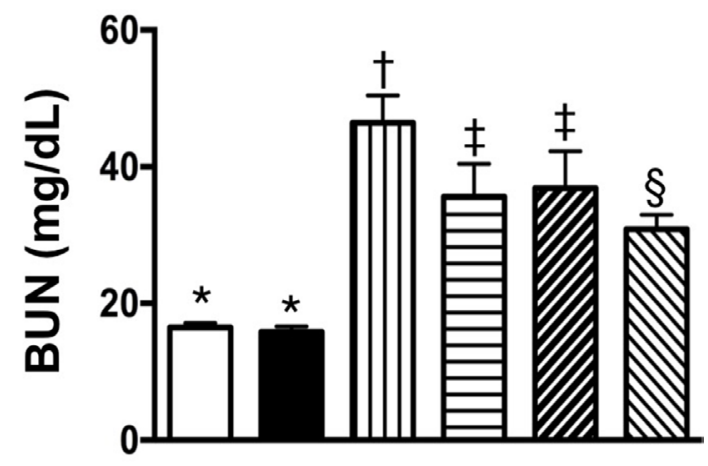

D

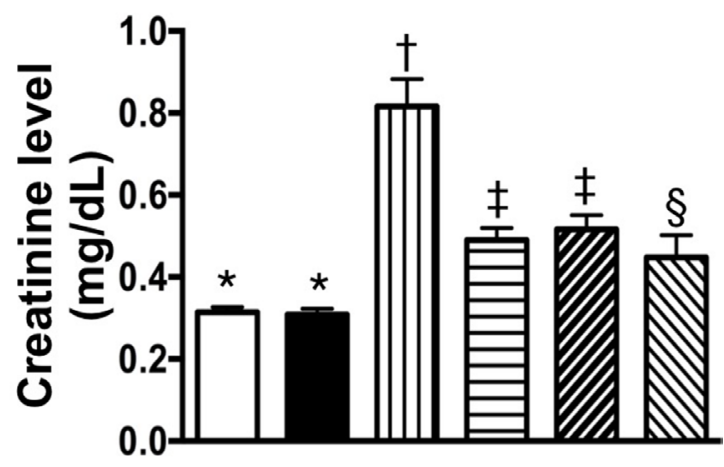

$\mathbf{F}$

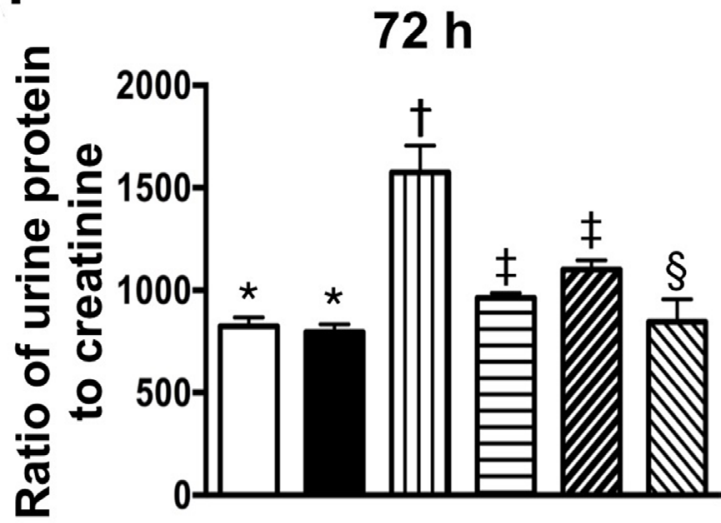

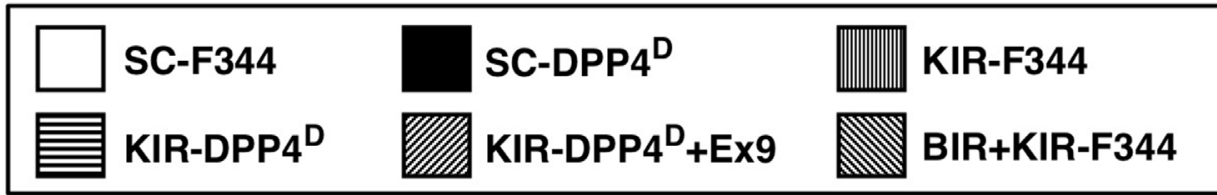

Figure 1: Blood urea nitrogen (BUN) and creatinine levels and urine protein to creatinine ratios at days 0 and 3 after acute KIR. (A, B) Circulating levels of blood urine nitrogen (BUN) at day $0(p>0.5)$ and day 3 after acute KIR in the 6 groups of rats. $(\mathbf{C}, \mathbf{D})$ Circulating level of creatinine at day $0(p>0.5)$ and day 3 after acute KIR in the 6 groups of rats. $(\mathbf{E}, \mathbf{F})$ Urine protein to creatinine ratio on day $0(p>0.5)$ and day 3 after acute KIR in the 6 groups of rats. Note: *denotes statistical significance vs. other groups with different symbols $(\dagger, \ddagger, \S) ; p<0.0001$. All statistical analyses were performed by one-way ANOVA and Bonferroni multiple comparison post-hoc test $\left(n=8\right.$ for each group). Symbols $(*, \dagger, \ddagger, \S)$ indicate statistical significance. $\mathrm{SC}=$ sham control; DPP4 ${ }^{\mathrm{D}}=$ dipeptidyl peptidase 4 deficient; $\mathrm{BIR}=$ small bowel ischemia-reperfusion; $\mathrm{KIR}=$ kidney ischemia-reperfusion. 
Status of inflammation, apoptosis and oxidative-stress on day 3 after acute KIR

Further, we analyzed the status of inflammation in the 6 groups of mice by analyzing the expression of matrix metalloproteinase (MMP)-9, tumor necrosis factor (TNF)- $\alpha$, nuclear factor (NF)- $\kappa B$, interleukin (IL)- $1 \beta$, intercellular adhesion molecule (ICAM)-1, and inducible nitric oxide synthase (iNOS). We observed that these six indicators of inflammation were lowest in SC-F344 and SC-DPP4 $4^{\mathrm{D}}$ rats and gradually increased in BIR-KIR-F344, KIR-DPP4 ${ }^{\mathrm{D}}$, DPP4 ${ }^{\mathrm{D}}$-KIR- exendin-9-39 rats and were highest in KIR-F344 rats (Figure 3).

A similar pattern was observed for the 3 apoptotic indicators, namely cleaved caspase 3 , cleaved poly (ADPribose) polymerase (c-PARP) and mitochondrial-Bax, and $\gamma-\mathrm{H} 2 \mathrm{AX}$ that is a marker for DNA damage and NOX1, NOX2, and total oxidized protein that indicate oxidative stress (Figure 4).
Status of podocyte components, antioxidants and GLP-R on day 3 after acute KIR

We observed that zonula occludens 1 (ZO-1), a tight junction-associated protein in podocytes, and P-cadherin and E-cadherin, which are predominantly in renal glomerulus and endothelial nitric oxide synthase (eNOS), an indicator of endothelial function, vasorelaxation integrity and angiogenesis were high in SC-F344 and SCDPP4 ${ }^{\mathrm{D}}$ rats and progressively lower in BIR-KIR-F344, KIR-DPP4 ${ }^{\mathrm{D}}$, DPP4 $4^{\mathrm{D}}-\mathrm{KIR}$ - exendin-9-39 rats and were lowest in KIR-F344 rats (Figure 5). The levels of GLP-1R and 3 antioxidant proteins, NQO 1, HO-1 and SOD-1 progressively increased from groups 1-6 suggesting an intrinsic response to ischemic stimulation and further upregulation due to DPP4 deficiency and BIR. GLP-1R levels were higher in DPP4 ${ }^{\mathrm{D}}$-KIR- exendin-9-39 rats compared to KIR-DPP4 ${ }^{\mathrm{D}}$ suggesting that exendin-9-39 treatment resulted in a competition with GLP-1,
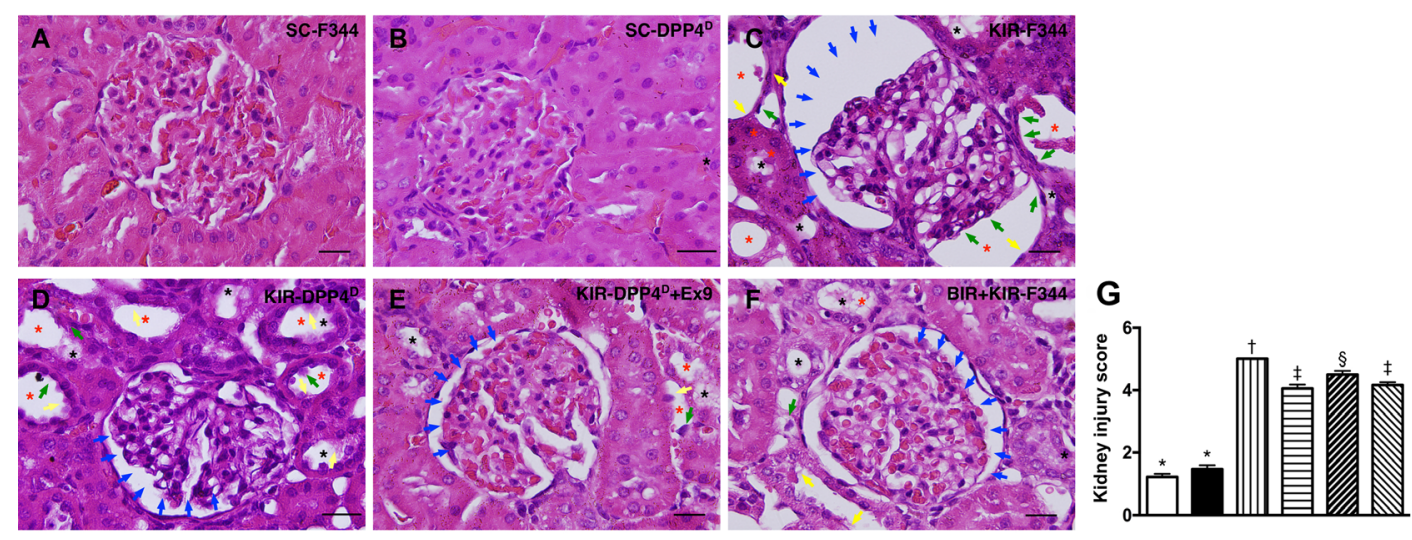

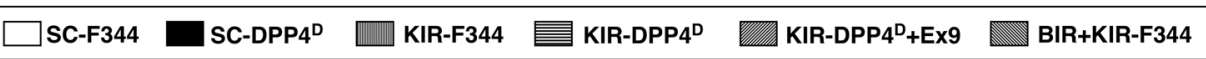
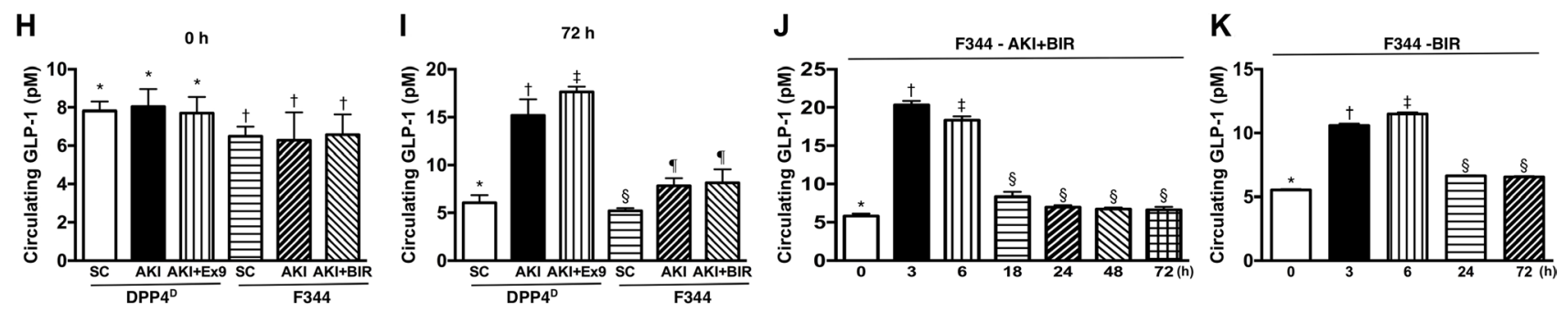

Figure 2: Kidney injury scores and circulating levels of GLP-1 at different time points after acute KIR. (A-F) Representative H\&E stained kidney sections $(200 \times)$ of the 6 groups of experimental rats demonstrating higher loss of brush border in renal tubules (yellow arrows), tubular necrosis (green arrows), tubular dilation (red asterisk), protein cast formation (black asterisk), and dilation of Bowman's capsule (blue arrows). (G) Kidney injury scores based on pathological analysis based on H\&E sections of the 6 groups of experimental rats is shown. Note: * denotes statistical significance vs. other groups with different symbols $(\dagger, \ddagger, \S), p<0.0001$. Scale bars in right lower corner represent $20 \mu \mathrm{m}$. (H) Baseline (day 0) circulating levels of GLP-1 in the 6 rat groups; * denotes statistical significance vs. ${ }^{\dagger} p<0.05$. (I) Circulating levels of GLP-1 in KIR-DPP4 ${ }^{\mathrm{D}}$ and KIR-F344 group of rats on day 3 after KIR. * denotes statistical significance vs. other groups with different symbols $(\dagger, \ddagger), p<0.0001 ; \S$ denotes statistical significance vs. ${ }^{q} p<0.01$. (J) Circulating levels of GLP1 at different time points in BIR-KIR-F344 animals. * denotes statistical significance vs. other groups with different symbols $(\dagger, \ddagger$, $\S)$; $p<0.0001$. (K) Circulating levels of GLP-1 at different time points in BIR-F344 animals. *denotes statistical significance vs. other groups with different symbols $(\dagger,+, \S) ; p<0.0001$. 
thereby enhancing the expression of GLP-1R in kidney parenchyma.

Status of infiltrating inflammatory cells in kidney parenchyma on day 3 after acute KIR

Immunofluorescence (IF) of kidney sections showed low numbers of $\mathrm{CD} 14^{+}$and $\mathrm{F} 4 / 80^{+}$cells, two indicators of inflammation in SC-F344 and SC-DPP4 ${ }^{\mathrm{D}}$ rats and gradually higher in BIR-KIR-F344, KIR-DPP4 ${ }^{\mathrm{D}}$, DPP4 $4_{-}$ KIR- exendin-9-39 rats and were highest in KIR-F344 rats (Figure 6).

\section{Status of cellular oxidative stress and kidney injury on day 3 after acute KIR}

The SC-F344 and SC-DPP4 ${ }^{\mathrm{D}}$ rats showed lower numbers of $\mathrm{H}_{2} \mathrm{DCFDA}^{+}$cells in the kidney parenchyma, an indicator of ROS, whereas they gradually increased in
BIR-KIR-F344, KIR-DPP4 ${ }^{\text {, }}$, DPP4D-KIR- exendin-9-39 rats and were highest in KIR-F344 rats (Figure 7). The expression pattern of KIM-1, a kidney injury biomarker that is predominantly expressed in renal tubules was similar to H2DCFDA in the six rat groups.

\section{Status of podocyte, extracellular-matrix and renal tubule components on day 3 after acute KIR}

Both ZO-1, a tight junction-associated protein in podocytes and Fibronectin, which is involved in cellextracellular matrix interactions were highly expressed in SC-F344 and SC-DPP4 ${ }^{\mathrm{D}}$ rats and progressively lower in BIR-KIR-F344, KIR-DPP4 ${ }^{\text {, }}$, DPP4'-KIR- exendin-9-39 rats and were lowest in KIR-F344 rats (Figure 8). Fibronectin is situated in the mesangial and interstitial extracellular matrix and defective cell-extracellular matrix interactions contribute to ischemic renal failure [34].
A
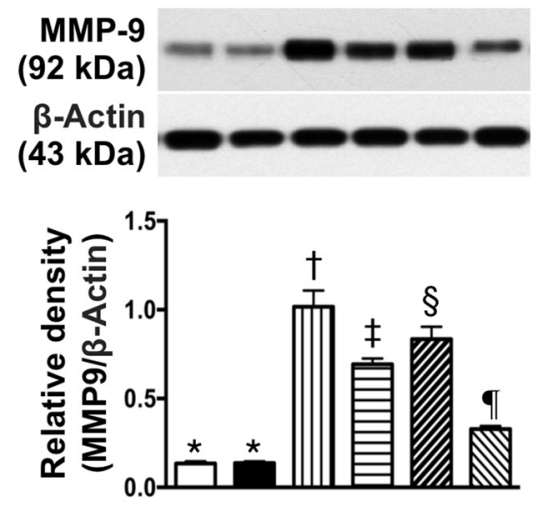

D
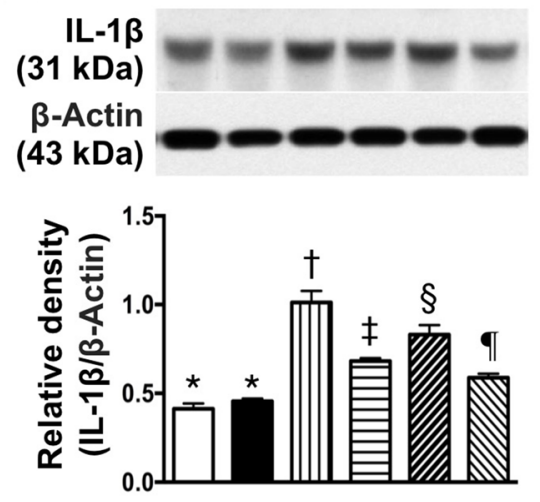

B
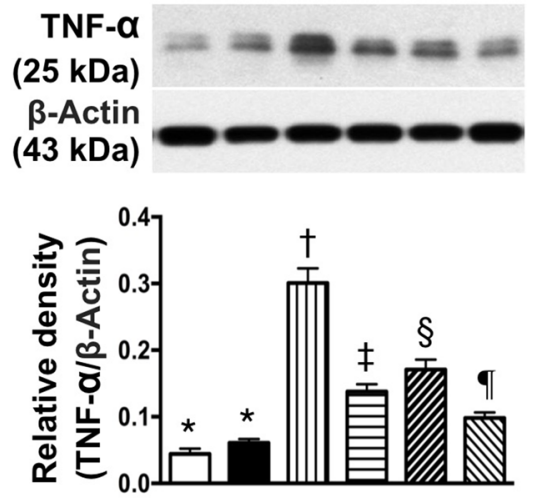

E
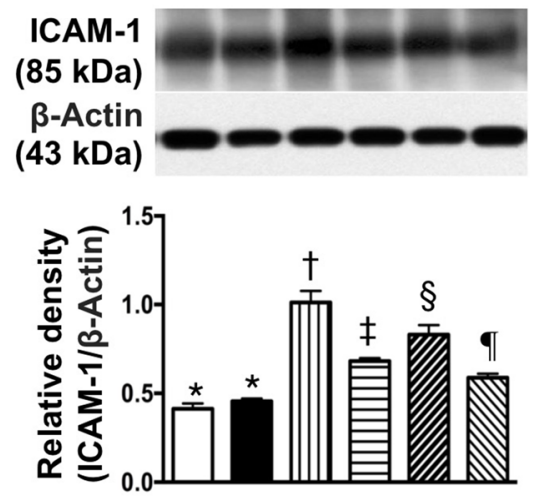

C $\mathrm{NF}-\mathrm{KB}$
$(65 \mathrm{kDa})$
$\beta-A c t i n$
$(43 \mathrm{kDa})$

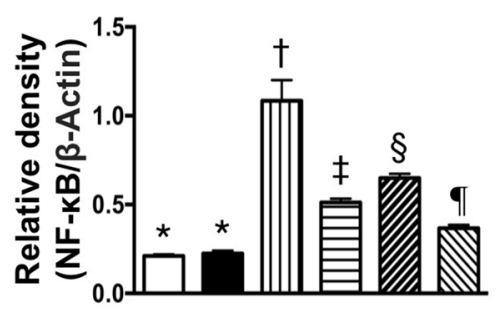

$\mathbf{F}$
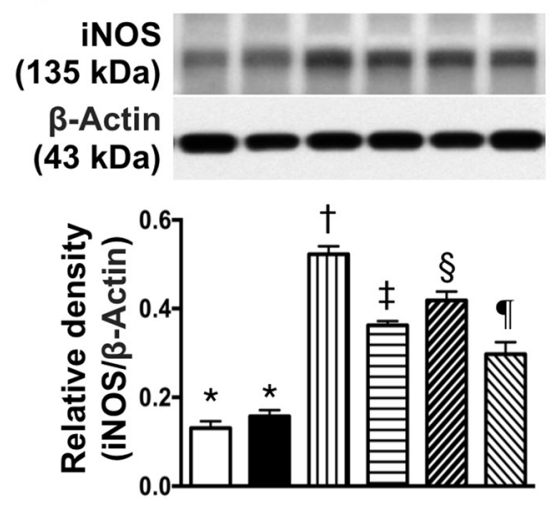

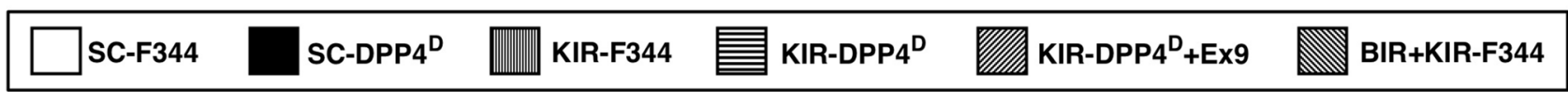

Figure 3: Analysis of kidney inflammation on day 3 after acute KIR. Western blotting analysis of (A) matrix metalloproteinase-9 (MMP-9), (B) tumor necrosis factor- $\alpha$ (TNF- $\alpha$ ), (C) Nuclear factor- $\kappa B$ (NFkB), (D) Interleukin-1 $\beta$ (IL-1 $\beta$ ), (E) Intercellular adhesion molecule-1 (ICAM-1), and (F) inducible nitric oxide synthase (iNOS) in the 6 groups of rats $72 \mathrm{~h}$ after acute KIR. Note: *denotes statistical significance vs. other groups represented with different symbols $(\dagger, \uparrow, \S, \mathbb{q}), p<0.0001$. 
We then analyzed the expression of P-cadherin and E-cadherin by IHC, which are $\mathrm{Ca}^{2+}$-dependent cell adhesion molecules involved in the development and maintenance of renal epithelial polarity. They showed an identical expression pattern to $\mathrm{ZO}-1$ among the six groups (Figure 9).

However, both FSP-1 and WT-1, which are predominantly expressed in kidney interstitials and podocytes, respectively showed opposite expression pattern to ZO-1 as analyzed by IHC (Figure 10).

\section{Status of dystroglycan and podocin expression on day 3 after acute KIR}

We demonstrated that dystroglycan, and podocin, two components of podocyte foot processes, were highly expressed in SC-F344 and SC-DPP4 ${ }^{\mathrm{D}}$ rats followed by progressively decreased levels in BIR-KIR-F344, KIRDPP4 ${ }^{\mathrm{D}}$, DPP4 ${ }^{\mathrm{D}-K I R}$ - exendin-9-39 rats and were lowest in KIR-F344 rats (Figure 11).

\section{DISCUSSION}

In this study, we investigated the role of DPP4 in acute KIR injury by using DPP knockout rats. We observed that baseline circulating GLP-1 levels were higher in DPP4 ${ }^{\mathrm{D}}$ rats compared to F344 rats. After KIR, GLP-1 levels were higher in the KIR-F344 group compared to SC-F344, but lower compared to KIR-DPP4 ${ }^{\mathrm{D}}$ and BIRKIR-F344 groups. This suggested that DPP4 deficiency and remote ischemic preconditioning protected kidneys against acute IR injury.

Previously, we showed that sitagliptin (DPP4 inhibitor) or exendin-4 (GLP-1 analogue) protected architectural integrity of the kidneys and renal function during acute KIR injury $[14,15]$. However, the role of DPP4 during acute kidney IR injury was not directly investigated, especially in regard to GLP-1 $[14,15]$. In this study, we showed that the circulating levels of creatinine and BUN and urine protein to creatinine ratio and anatomical/histopathological injury (H\&E, IHC and IF showing kidney morphology and kidney injury score) were substantially higher in KIR-F344 animals than in SC-F344 animals, but reduced in KIR-DPP4 ${ }^{\mathrm{D}}$ animals. This corroborated our previous findings that DPP4 influenced the extent of kidney damage during IR injury [14, 15]. These findings highlight the therapeutic potential of the DPP4 inhibitors and GLP-1 analogues during AKI, especially in type 2 DM patients.

Remote ischemic preconditioning has been shown to protect other organs/tissues from IR injury [29-31]. Remote ischemic preconditioning involves complex signaling and interactions that activate ATP-dependent potassium channels, adenosine and $\mathrm{PKC}$, release
A
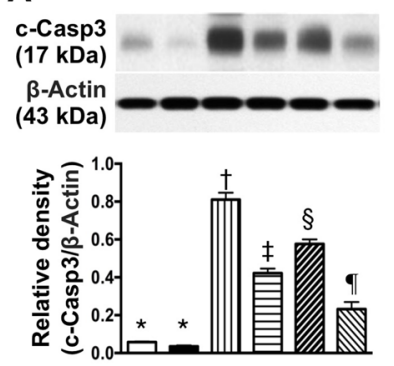

D
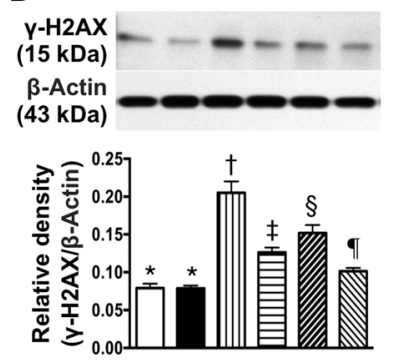

B
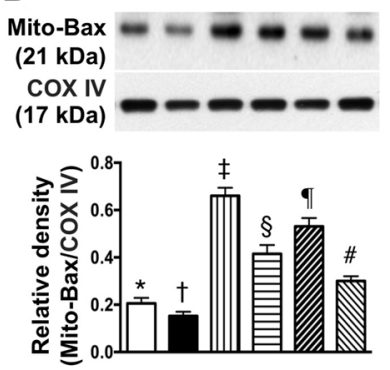

(E
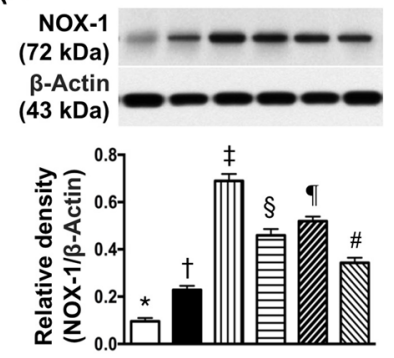

C

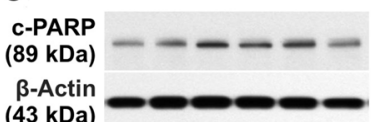

(43 kDa)

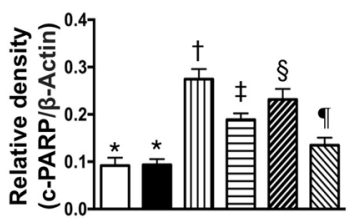

$\mathbf{F}$
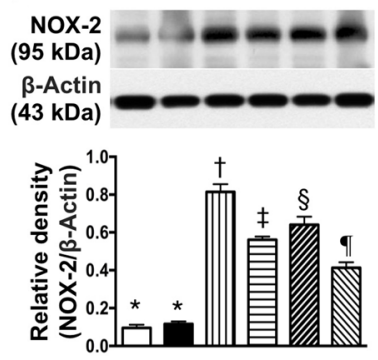

G
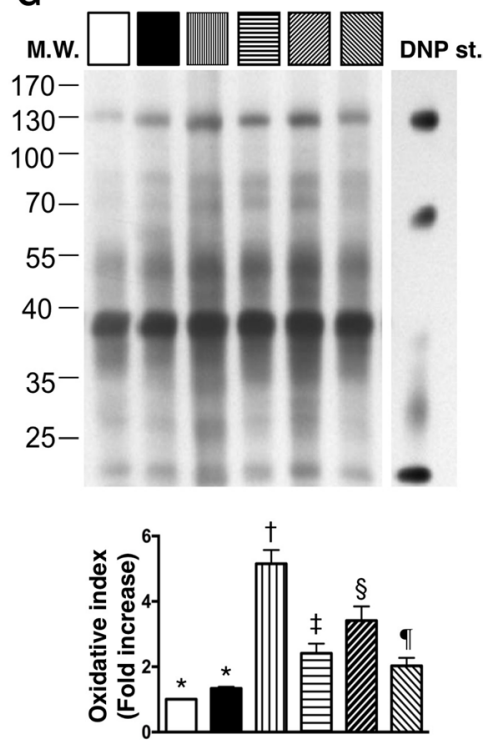

Figure 4: Analysis of apoptotic, DNA damage and oxidative stress in the kidney on day 3 after acute KIR. Western blot analysis of (A) cleaved caspase 3 (c-Casp3), (B) cleaved caspase 3 (c-Casp3), (C) cleaved Poly (ADP-ribose) polymerase (c-PARP), (D) $\gamma$-H2AX, (E) NOX-1 and (F) total oxidized protein by oxyblot (left most lanes in the upper panel represent protein molecular weight marker and control oxidized molecular protein standard, respectively; DNP is 1-3 dinitrophenylhydrazone) in the 6 groups of rats $72 \mathrm{~h}$ after acute KIR. Note *denotes statistical significance vs. other groups represented with different symbols $(\dagger, \ddagger, \S, \boldsymbol{q}), p<0.0001$. 
autacoids such as adenosine and bradykinin, generate neurohormones, nitric oxide and chemokines (such as hypoxia-inducible factor- $1 \alpha$ ), open mitochondrial $\mathrm{K}^{+}$ATP-channels and elicit GSK3 $\beta$ and STAT-3 signaling that attenuate opening of the mitochondrial permeability transition pore [29-31]. In this study, we show that remote ischemic preconditioning by small BIR protects kidneys against acute KIR injury. The renal function and kidney architectural integrity was better preserved in BIRKIR-F344 rats compared to KIR-DPP4 ${ }^{\mathrm{D}}$ rats.
The signal transduction pathways in ischemic pre-, post-, and remote conditioning that protect tissues/organs against acute IR are highly complex [30]. In the present study, circulating GLP-1 levels were higher in BIRKIR-F344 animals at 3 to $6 \mathrm{hrs}$ after acute KIR compared to the KIR-F344 group. Also, the circulating GLP-1 levels increased rapidly during the acute phase in BIR-KIR-F344 rats suggesting that the gastrointestinal-digestive tract was a major source of GLP-1 in response to ischemic stimulation as previously suggested [30].
A
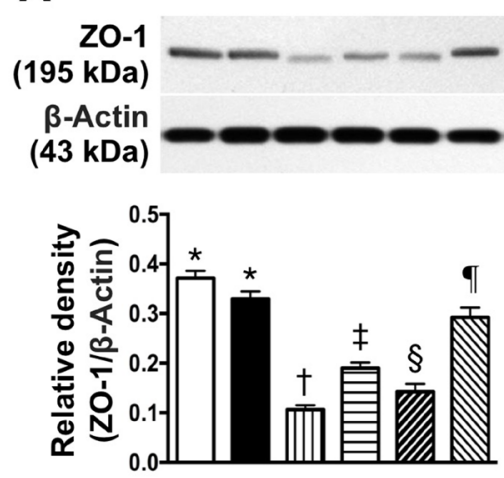

D
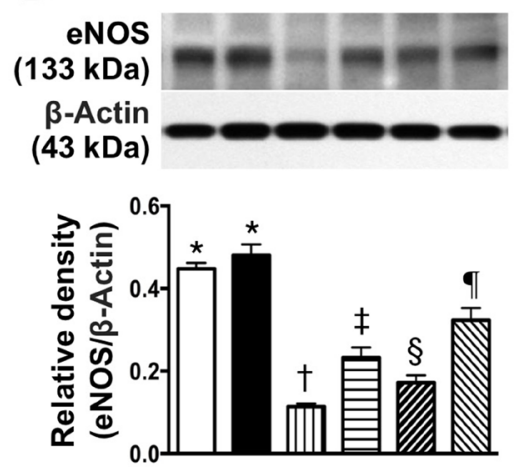

G
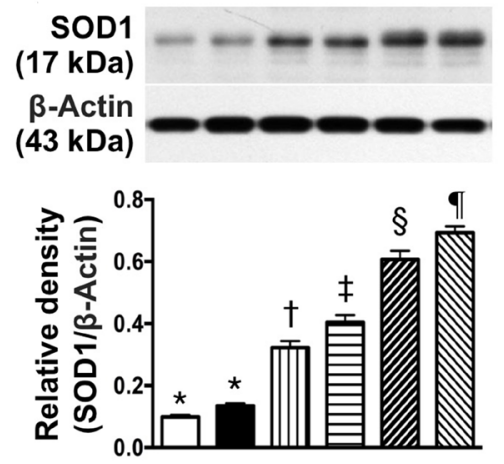

B
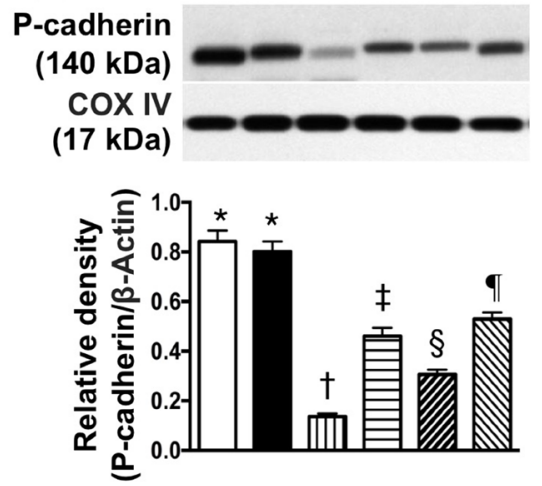

E
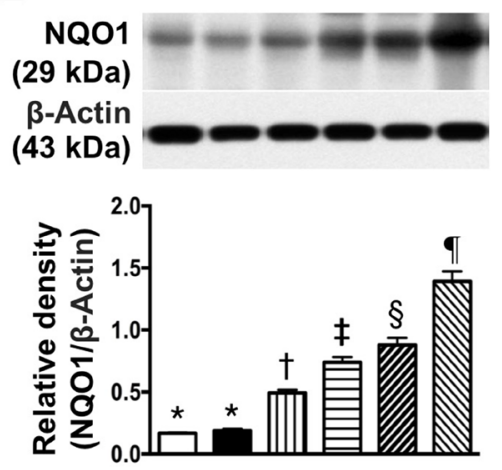

C
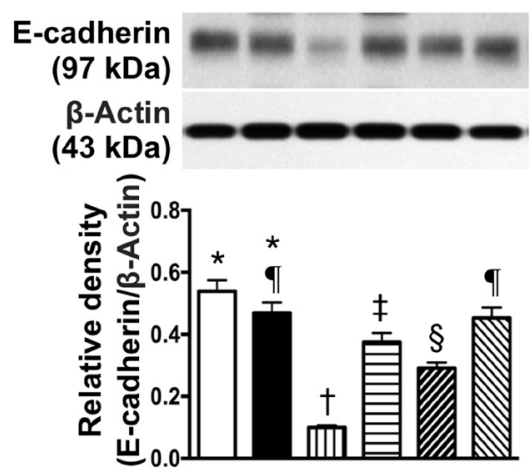

$\mathbf{F}$
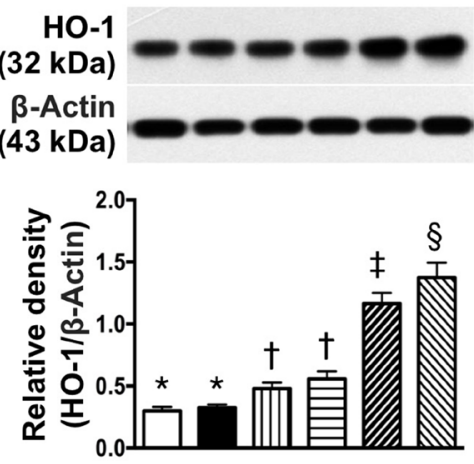

H
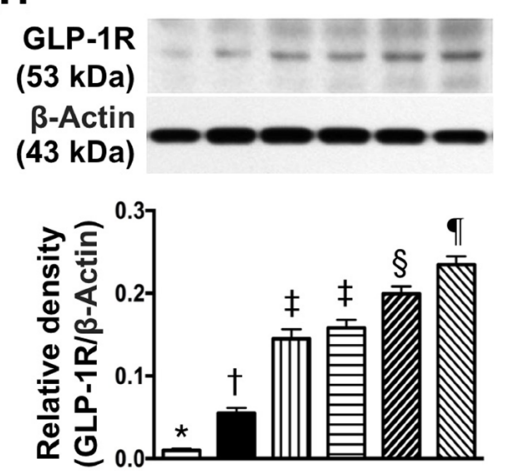

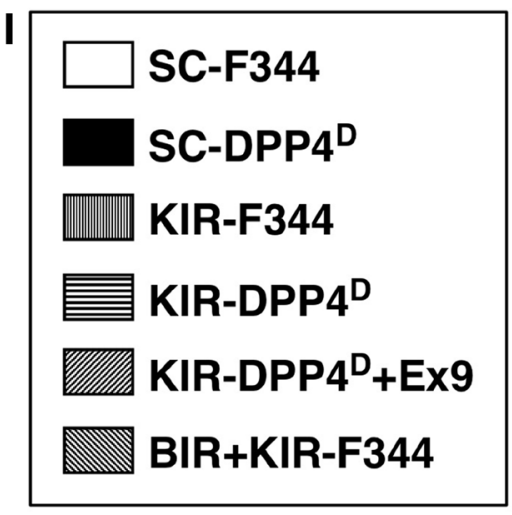

Figure 5: Analysis of podocyte components, antioxidants and GLP-R on day 3 after acute KIR. Western blot analysis of (A) ZO-1, (B) P-cadherin (C) E-cadherin (D) eNOS, (F) NQO1, (G) heme oxygenase 1 (HO-1), (H) SOD-1 and (I) glucagon like peptide receptor (GLP-R) in the 6 groups of rats $72 \mathrm{~h}$ after acute KIR. *denotes statistical significance vs. other groups represented with different symbols $(\dagger, \dagger, \S, \mathbb{q}), p<0.0001$. 
Numerous studies have demonstrated that AKI or acute KIR injury generates oxidative stress and reactive oxygen species [13-15], elicits cellular apoptosis [11, 13-15], and activates complement and inflammation [10-14, 27]. These factors damage the kidney function and its anatomical structure [13-15, 35]. In our study, the acute KIR rats showed increased inflammation, oxidative stress, DNA damage and apoptosis consistent with previous studies [10-15]. The extent of functional and architectural damage to the kidneys in KIR-F344 rats was significantly higher compared to SCF344, but lower in KIR-DPP4 ${ }^{\mathrm{D}}$ and BIR-KIR-F344 rats.

In a previous study, we demonstrated that acute KIR substantial damaged the kidney ultrastructure [15]. In this study, we confirmed that KIR substantially damaged the kidney ultrastructural integrity (components of podocyte foot process in glomeruli, renal tubule and extracellular matrix) in KIR-F344 rats compared to SC-F344 animals, but was better preserved in KIR-DPP4 ${ }^{\mathrm{D}}$ and BIRKIR-F344 rats. This suggested that DPP4 deficiency and remote ischemic preconditioning preserved renal function during acute kidney injury.

We also demonstrated increased expression of antioxidant proteins like NQO 1, HO-1 and SOD-1 and GLP-1 receptor in the kidney tissues of KIR-DPP4 ${ }^{D}$ and BIR-KIR-F344 rats compared to the sham controls and KIR-F344 rats. These findings suggested that DPP4 deficiency and remote ischemic preconditioning protected kidneys from the damage by ischemic stimulation and
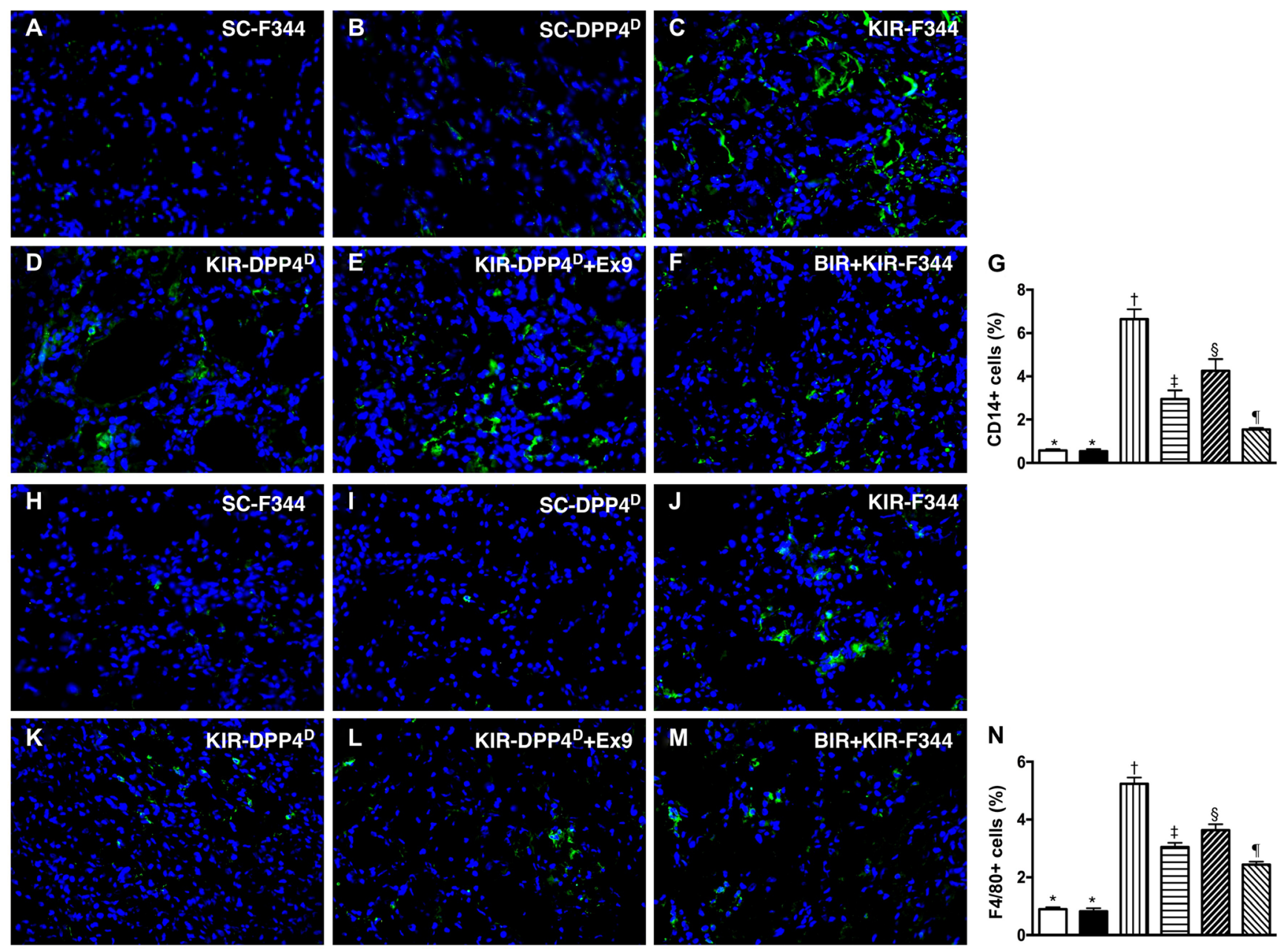

SC-F344

SC-DPP4 ${ }^{\mathrm{D}}$

KIR-F344 哣 KIR-DPP4

KIR-DPP4 ${ }^{\mathrm{D}}+\mathrm{Ex} 9 \mathbb{B I R + K I R - F 3 4 4}$

Figure 6: Analysis of infiltration of inflammatory cells in kidney parenchyma on day 3 after acute KIR. (A-F) Representative immunofluorescence (IF) images (400x) showing CD14 cells (green color) in the kidney parenchyma in the 6 groups of rats $72 \mathrm{~h}$ after acute KIR. (G) Quantification of mean total number of CD14+ cells in the kidney sections from 6 groups of rats, $72 \mathrm{~h}$ after acute KIR. (H-M) Representative IF images (400×) showing F4/80+ cells (green color) in kidney parenchyma in the 6 groups of rats $72 \mathrm{~h}$ after acute KIR. (N) Quantification of mean total number of F4/80+ cells in the kidney sections from 6 groups of rats, $72 \mathrm{~h}$ after acute KIR. Note: *denotes statistical significance vs. other groups represented with different symbols $(\dagger, \$, \S, \uparrow), p<0.0001$. Scale bars in the right lower corner represent $20 \mu \mathrm{m}$. Blue color indicates nuclei were stained by DAPI. 
IR injury. These findings support and improve our understanding of mechanisms that enable protective ischemic conditioning against acute IR injury in tissues/ organs [30].

This study has limitations that need to be highlighted. First, the study period was only $72 \mathrm{~h}$ and therefore long term impact of DPP4 deficiency and remote ischemic preconditioning were not addressed. Second, although the small BIR procedure was used to elicit remote ischemic preconditioning in rats and shown to protect the kidneys from IR injury, such a method is not currently feasible for human patients. Third, although we identified changes in many signaling and functional entities in response to acute kidney injury as well as their status in DPP4 deficient or remote ischemic preconditioning conditions, the exact interplay of the various pathways and their relationship to renal oxidative stress, inflammation or cellular apoptosis are still not clear. Figure 12 summarizes probable protective mechanisms in the DPP4 ${ }^{\mathrm{D}}$ rat kidneys and remote ischemic preconditioning against acute KIR. Fourth, although BIR was comparable to DPP4 deficiency in protecting the kidney from acute IR injury, it wasn't clear if increased GLP-1 mediated the protective effects of BIR.

In conclusion, our study demonstrates that DPP4 deficiency and remote ischemic preconditioning protect kidneys from acute IR injury by elevating circulating GLP-1 levels and represent potential therapeutic targets
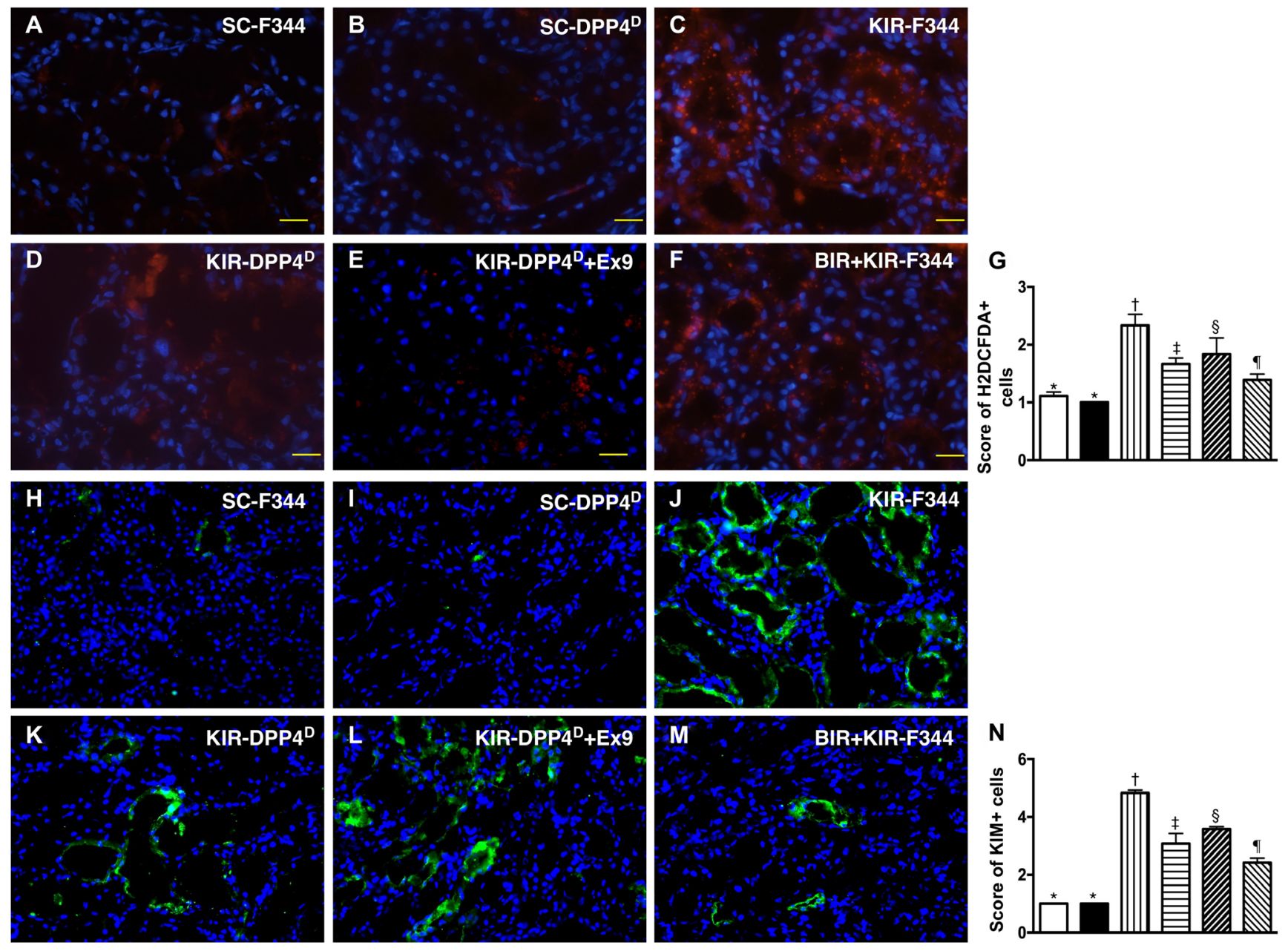

SC-F344

SC-DPP4 ${ }^{\mathrm{D}}$

KIR-F344 点 KIR-DPP4 ${ }^{\mathrm{D}}$

KIR-DPP4 ${ }^{\mathrm{D}}+\mathrm{Ex9} \mathbb{B}$ BIR+KIR-F344

Figure 7: Analysis of cellular oxidative stress and kidney injury markers on day 3 after acute KIR. (A-F) Representative IF images $(200 \times)$ showing H2DCFDA ${ }^{+}$cells (red color) in the kidney parenchyma of the 6 groups of rats, $72 \mathrm{~h}$ after acute KIR. (G) Quantification of total number of $\mathrm{H}_{2} \mathrm{DCFDA}^{+}$cells in the kidney sections from 6 groups of rats, $72 \mathrm{~h}$ after acute KIR. *denotes statistical significance vs. other groups represented with different symbols $(\dagger, \downarrow, \S, \mathbb{q}), p<0.0001$. Scale bars in right lower corner represent $50 \mu \mathrm{m}$. $(\mathbf{H}-\mathbf{M})$ Representative IF images (400×) showing $\mathrm{KIM}^{+}$- cells (green color). (N) Quantification of mean total number of KIM+ cells in the kidney sections from 6 groups of rats, $72 \mathrm{~h}$ after acute KIR. *denotes statistical significance vs. other groups represented with different symbols $(\dagger, \ddagger, \S, \uparrow), p<0.0001$. Scale bars in right lower corner represent $20 \mu \mathrm{m}$. Blue color indicates DAPI stained nuclei. 
for acute kidney injury, especially in type 2 diabetic patients.

\section{MATERIALS AND METHODS}

\section{Experimental animals}

The animal experiments were conducted according to approved protocols by the Animal Care and Use Committee at Kaohsiung Chang Gung Memorial Hospital (Affidavit of Approval of Animal Use Protocol No. 2015032402) and executed according to the Guide for the Care and Use of Laboratory Animals, 8th edition (NRC 2011). Pathogen-free, adult male $(n=32)$ and dipeptidyl peptidase 4 (DPP4) mutant $(n=16)$ Fischer 344 rats weighing 300-320 g were obtained from Charles River Technology (BioLASCO Taiwan Co. Ltd., Taiwan). The animals were housed in an AAALAC-approved animal facility in our hospital with controlled temperature and light cycles $\left(24^{\circ} \mathrm{C}\right.$ and $12 / 12 \mathrm{~h}$ light/dark cycles $)$.

\section{Acute kidney ischemia-reperfusion (IR) protocol and animal grouping}

The acute kidney IR was performed according to previously published protocol $[15,35]$. Briefly, animals were anesthetized with $2 \%$ isoflurane and placed supine on a warming pad at $37^{\circ} \mathrm{C}$ for midline laparotomies.
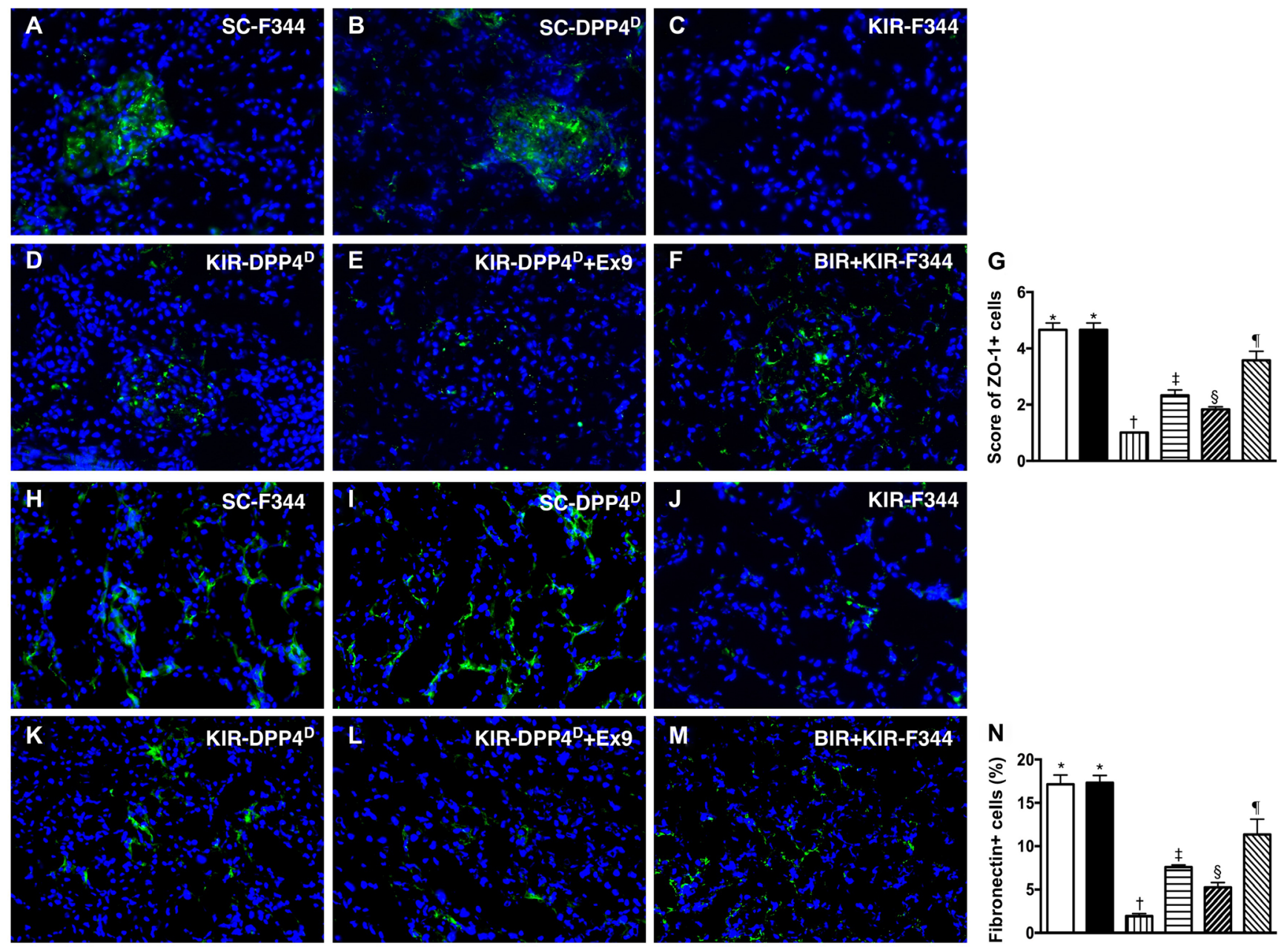

\section{SC-F344}

SC-DPP4

KIR-F344 馬 KIR-DPP4

KIR-DPP4 ${ }^{\mathrm{D}}+\mathrm{Ex} 9$

BIR+KIR-F344

Figure 8: Immunofluorescence (IF) analysis of ZO-1 and fibronectin expression on day 3 after acute KIR. (A-F) Representative IF images (400×) showing positively stained ZO-1 (green color) distribution in glomeruli of the 6 groups of rats, $72 \mathrm{~h}$ after acute KIR. (G) Quantification of total number of ZO-1 positive cells in the kidneys of 6 groups of rats, $72 \mathrm{~h}$ after acute KIR. (H-M) Representative IF images (400×) showing positively stained fibronectin (green color) distribution in extracellular matrix of 6 groups of rats, $72 \mathrm{~h}$ after acute KIR. (N) Quantification of total number of fibronectin positive cells in kidneys of the 6 groups of rats, $72 \mathrm{~h}$ after acute KIR. *denotes statistical significance vs. other groups represented with different symbols $(\dagger, \ddagger, \S, \mathbb{\uparrow}), p<0.0001$. Scale bars in right lower corner represent $20 \mu \mathrm{m}$. Blue color indicates DAPI stained nuclei. 
The sham control (SC) animals underwent laparotomy only. Acute IR injury of both kidneys was induced by clamping the renal pedicles of the rats with non-traumatic vascular clips for 60 minutes followed by reperfusion for $72 \mathrm{~h}$. The animals were then euthanized and the kidneys were harvested for further experiments. Left kidney was used for H\&E staining, immunofluorescence (IF) and immunohistochemistry (IHC) and the right kidney was used for western blotting.

The rats were categorized into six groups $(n=8$ group): (1) sham control F344 (SC-F344) with laparotomy without kidney IR (KIR); (2) sham control DPP4 mutant rats (SC-DPP4D); (3) kidney IR in F344 rats (KIR-F344); (4) kidney IR in DPP4 mutant rats (KIR-DPP4 $)$; (5)
KIR-DPP4 ${ }^{\mathrm{D}}$ with intraperitoneal injection of $10 \mathrm{mg} / \mathrm{kg}$ exendin-9-39 at 30 minutes and days 1-3 after KIR; and (6) small bowel IR in F344 rats with KIR (BIRKIR-F344). Exendin-9-39 is an antagonist of exendin 4, a hypoglycemic agent and a GLP-1 analog used for type II diabetes mellitus. The dosage of exendin-9-39 was based on our previous study [14].

\section{Remote ischemic preconditioning by intermittent small bowel IR}

The small bowel IR injury was performed according to previously published protocol [36]. Briefly, the small BIR injury in group 6 rats involved clamping the superior
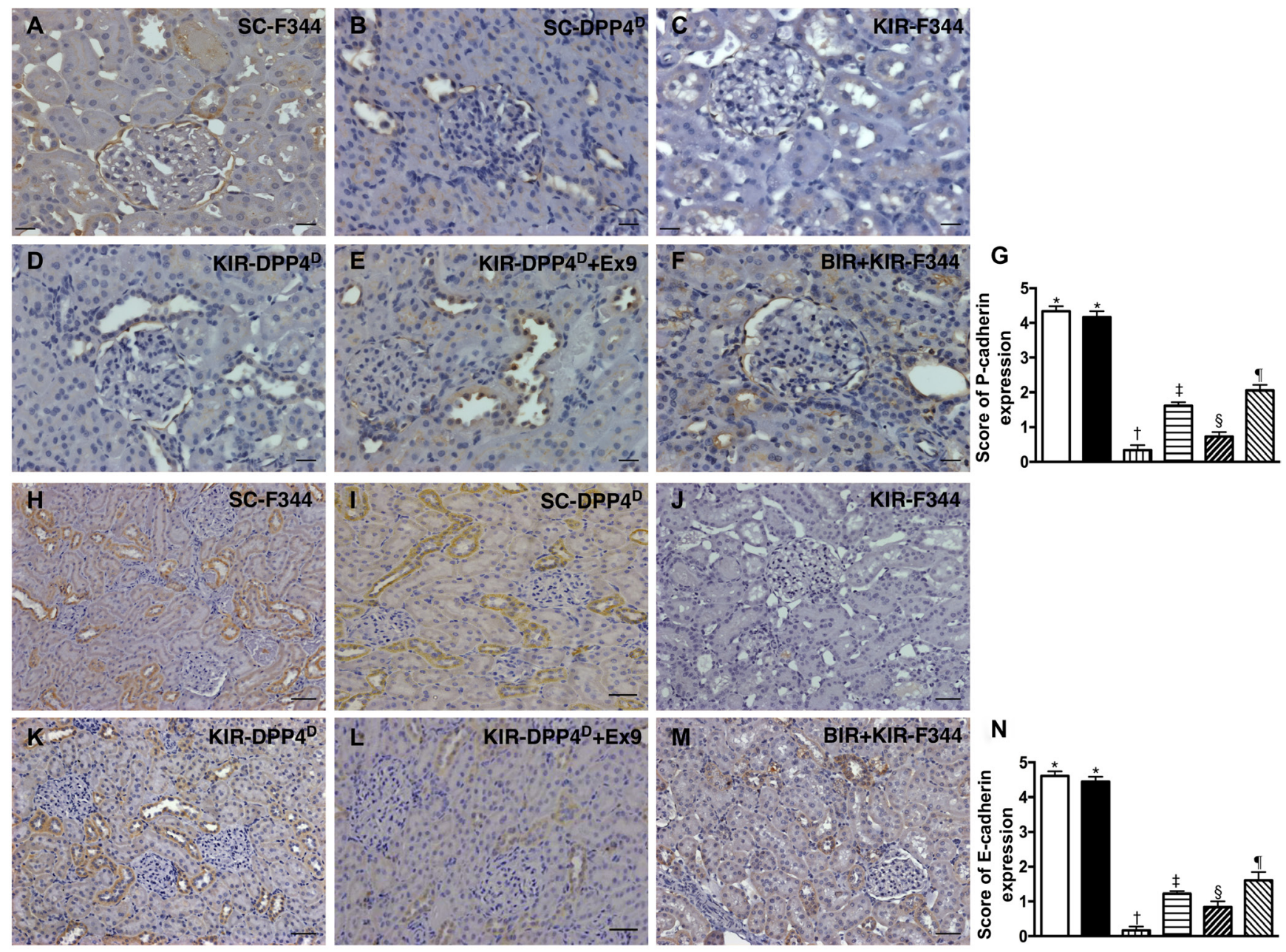

SC-F344

SC-DPP4 ${ }^{\mathrm{D}}$

KIR-F344 腎 KIR-DPP4 ${ }^{\text {D }}$

KIR-DPP4 ${ }^{\mathrm{D}}+\mathrm{Ex} 9$

BIR+KIR-F344

Figure 9: Immunohistochemical (IHC) analysis of P-cadherin and E-cadherin expression on day 3 after acute KIR. (A-F) Representative IHC images (200×) showing positively stained P-cadherin (gray color) distribution in renal tubules of the 6 groups of rats, $72 \mathrm{~h}$ after acute KIR. (G) Quantification of total number of P-cadherin positive cells in the kidney sections from 6 groups of rats, $72 \mathrm{~h}$ after acute KIR injury. (H-M) Representative IHC images (200×) showing positively stained E-cadherin (gray color) distribution in extracellular matrix of 6 groups of rats, $72 \mathrm{~h}$ after acute KIR. (N) Quantification of total number of E-cadherin positive stained cells in the kidney sections from 6 groups of rats, $72 \mathrm{~h}$ after acute KIR. *denotes statistical significance vs. other groups represented with different symbols $(\dagger, \ddagger, \S, \Phi), p<0.0001$. Scale bars in right lower corner represent $50 \mu \mathrm{m}$ in the kidney sections from 6 groups of rats, $72 \mathrm{~h}$ after acute KIR injury. 
mesenteric artery for 5 mins followed by reperfusion for 5 mins and repeating the procedure 6 times. The acute kidney IR procedure was performed immediately after finishing the small BIR procedure.

\section{Estimating kidney injury scores after acute KIR}

Histopathology scoring was performed on kidney samples isolated on day 3 after KIR and were assessed blind as previously described [13-15]. Briefly, the kidney specimens were fixed in $10 \%$ buffered formalin and embedded in paraffin. Then, $5 \mu \mathrm{m}$ sections were cut and stained with hematoxylin and eosin (H\&E) and observed by light microscopy. The scoring system involved grading the extent of tubular necrosis, loss of brush border, cast formation and tubular dilation in 10 randomly chosen non-overlapping fields $(200 \times)$ for each rat kidney sample [32]. The scores were $0(0 \%), 1(\leq 10 \%), 2(11-25 \%)$, $3(26-45 \%), 4(46-75 \%)$, and $5(\geq 76 \%)$.

\section{Estimation of blood urea nitrogen and creatinine and urine protein to creatinine ratio after acute KIR}

Blood samples were collected from the rats in all 6 groups before and $72 \mathrm{~h}$ after IR to measure changes in serum creatinine and urea nitrogen.

To determine the ratio of urine protein to creatinine, each animal was placed in a metabolic cage (DXL-D, $190 \mathrm{~mm} \times 290 \mathrm{~mm} \times 550 \mathrm{~mm}$, Suzhou Fengshi Laboratory
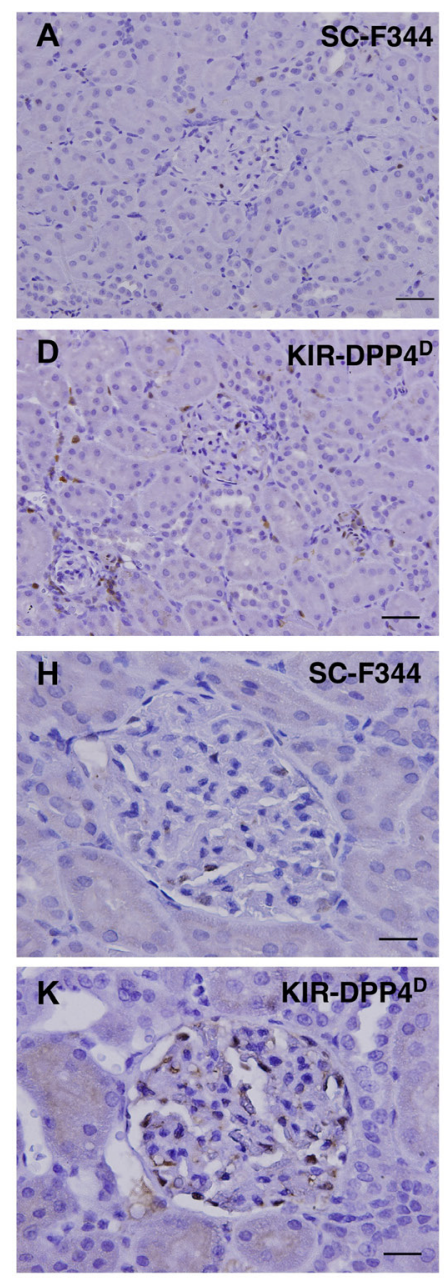

SC-F344
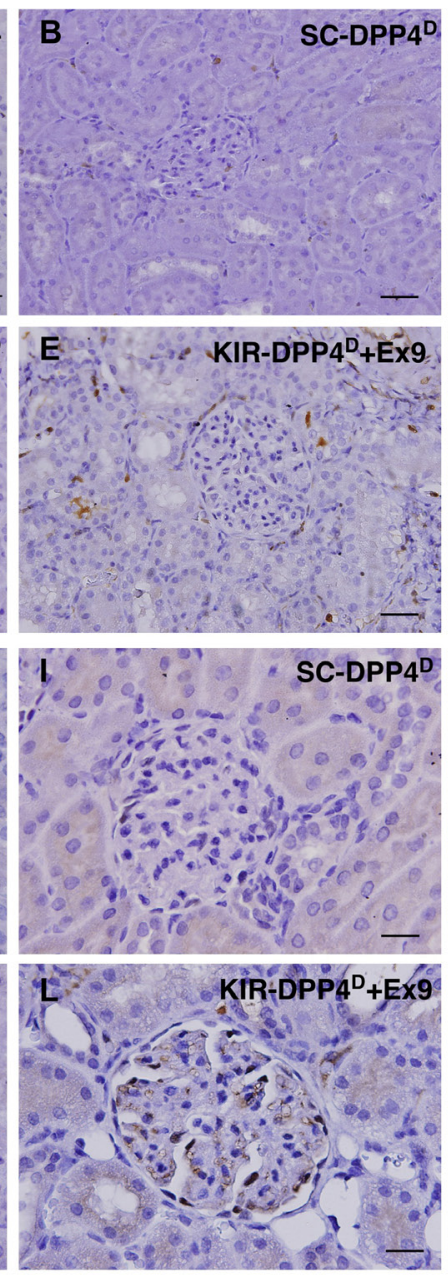
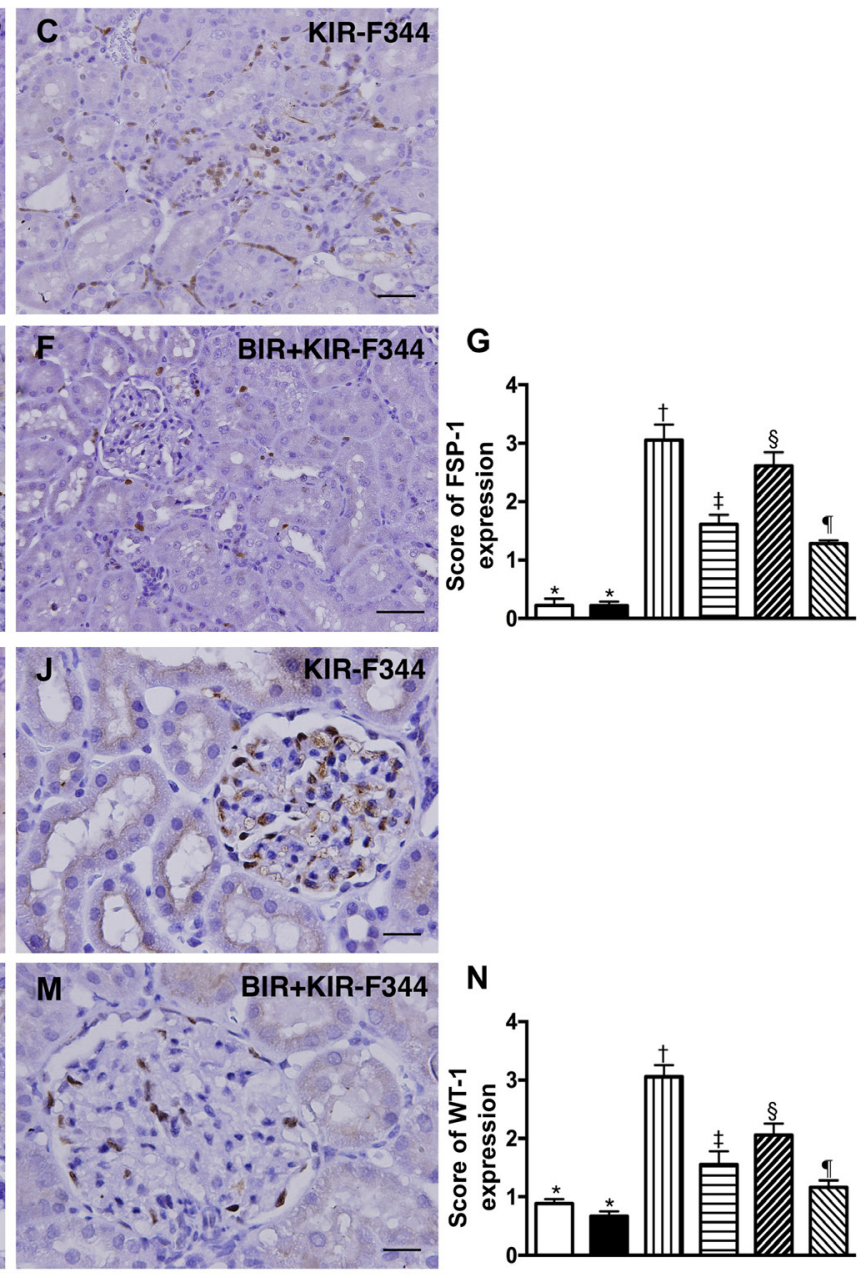

Figure 10: Immunohistochemical (IHC) analysis of FSP-1 and WT-1 expression on day 3 after acute KIR. (A-F) Representative IHC images (200×) showing positively stained fibroblast specific protein-1 (FSP-1) (gray color) distribution in kidney interstitials from 6 groups of rats, $72 \mathrm{~h}$ after acute KIR injury. (G) Quantification of total number of FSP-1 positive cells in the kidney sections from 6 groups of rats, $72 \mathrm{~h}$ after acute KIR. (H-M) Representative IHC images $(200 \times)$ for positively stained Wilm's tumor suppressor gene1 (WT-1) (gray color) in podocytes from 6 groups of rats, $72 \mathrm{~h}$ after acute KIR. (N) Quantification of total number of WT-1 positive cells in the kidney sections from 6 groups of rats, $72 \mathrm{~h}$ after acute KIR. *denotes statistical significance vs. other groups represented with different symbols $(\dagger,+, \S, \mathbb{q}), p<0.0001$. Scale bars in right lower corner represent $50 \mu \mathrm{m}$. 
Animal Equipment Co. Ltd., Mainland China) for $24 \mathrm{~h}$ with free access to food and water. Urine was collected for $24 \mathrm{~h}$ before and $72 \mathrm{~h}$ after IR.

\section{IHC and IF staining}

The IHC and IF staining was performed according to previously published protocols [13-15]. For IHC and IF staining, rehydrated paraffin sections were treated with $3 \% \mathrm{H}_{2} \mathrm{O}_{2}$ for 30 minutes and then incubated with Immuno-Block reagent (BioSB, Santa Barbara, CA, USA) for 30 minutes at room temperature. Then, the sections were incubated with the following primary antibodies: zonula occludens-1 (ZO-1; 1:300, Abcam, Cambridge, MA, USA), Wilm's tumor suppressor gene 1 (WT-1;
1:1000, Abcam, Cambridge, MA, USA), kidney injury molecule (KIM-1;1:200, R\&D systems, Minneapolis, MN, USA), fibroblast specific protein (FSP-1; 1:200, Abcam, Cambridge, MA, USA), P-cadherin (1:100, Novus, Littleton, CO, USA), podocin (1:100,Sigma, St. Louis, Mo, USA), dystrophin (1:100, Abcam, Cambridge, MA, USA), fibronectin (1:200, Abcam, Cambridge, MA, USA), dystroglycan (1:50, Abcam, Cambridge, MA, USA), synaptopodin (1:100, Santa Cruz, CA, USA), CD14 (1:50, Santa Cruz, CA, USA), CD68 (1:100, Abcam, Cambridge, MA, USA); sections incubated with irrelevant antibodies served as controls. Three kidney sections were analyzed from each rat. For quantification, three randomly selected high power fields (HPFs; $200 \times$ for IHC; $400 \times$ for IF) were analyzed in each section. The mean number of positively-
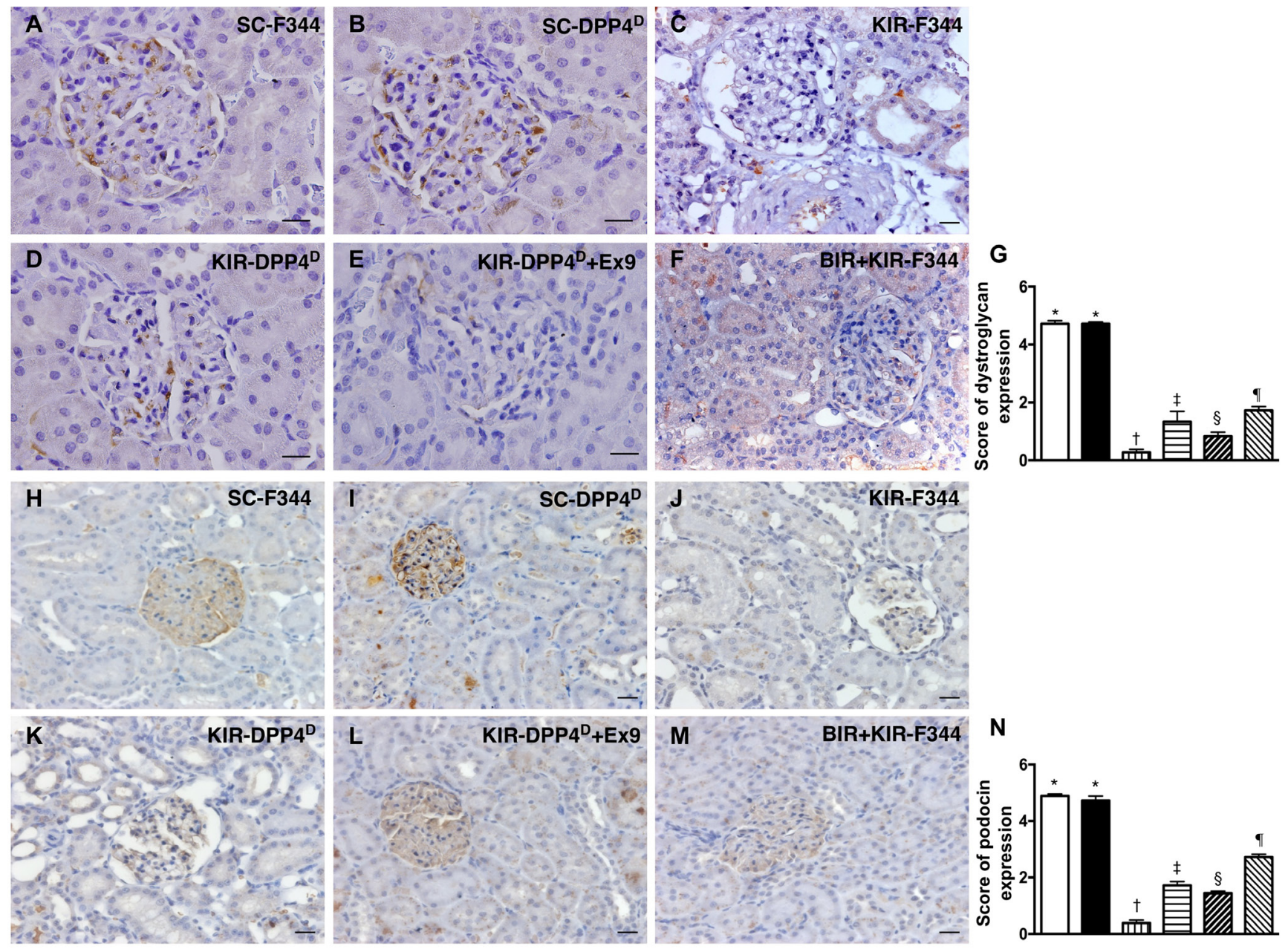

SC-F344

SC-DPP4

KIR-F344

KIR-DPP4

KIR-DPP4 ${ }^{\mathrm{D}}+\mathrm{Ex} 9$

BIR+KIR-F344

Figure 11: IHC analysis of dystroglycan and podocin on day 3 after acute KIR. (A-F) Representative IHC images (400×) for positively stained dystroglycan (gray color) in podocyte foot process from 6 groups of rats, $72 \mathrm{~h}$ after acute KIR. (G) Quantification of total number of dystroglycan positive cells. (H-M) Representative IHC images $(200 \times)$ for positively stained podocin (gray color) in podocytes from 6 groups of rats, $72 \mathrm{~h}$ after acute KIR. (N) Quantification of total number of podocin positive cells in kidney sections from 6 groups of rats, $72 \mathrm{~h}$ after acute KIR. *denotes statistical significance vs. other groups represented with different symbols $(\dagger, \ddagger$, $\S, \mathbb{q}), p<0.0001$. Scale bars in right lower corner represent $50 \mu \mathrm{m}$. 
stained cells per HPF for each animal was determined by summation of all numbers divided by 9 .

\section{Western blot analysis}

Western blot analysis was performed according to previous published protocols [13-15]. Briefly, $50 \mu \mathrm{g}$ of total kidney protein extract from different groups of rats were separated by SDS-PAGE using acrylamide gradients. Then, the separated proteins were transferred electrophoretically to a polyvinylidene difluoride (PVDF) membrane (Amersham Biosciences, Amersham, UK). Non-specific binding was blocked by incubating the membrane in 5\% nonfat dry milk in T-TBS (TBS containing 0.05\% Tween 20) overnight. Then, the membranes were incubated for $1 \mathrm{~h}$ at room temperature with primary antibodies against matrix metalloproteinase (MMP-9; 1:3000, Abcam, Cambridge,
MA, USA), tumor necrosis factor (TNF- $\alpha ; 1: 1000$, Cell Signaling, Danvers, MA, USA), nuclear factor (NF-кB; 1:600, Abcam, Cambridge, MA, USA), interleukin (IL-1 $\beta ; 1: 1000$, Cell Signaling, Danvers, MA, USA), intercellular adhesion molecule (ICAM-1; 1:1000, Abcam, Cambridge, MA, USA), inducible nitric oxide synthase (iNOS; 1:200, Abcam, Cambridge, MA, USA), cleaved caspase 3 (1:1000, Cell Signaling, Danvers, MA, USA), cleaved PolyADP-ribosepolymerase (c-PARP; 1:1000, Cell Signaling, Danvers, MA, USA), mitochondrial Bax (1:1000, Abcam, Cambridge, MA, USA), $\gamma$-H2AX (1:1000, Cell Signaling, Danvers, MA, USA), endothelial nitric oxide synthase (eNOS; 1:1000, Abcam, Cambridge, MA, USA), heme oxygenase-1 (HO-1; 1:500, Cell Signaling, Danvers, MA, USA), glucagon like peptide 1 receptor (GLP-1R; 1:1000, Abcam, Cambridge, MA, USA), superoxide dismutase (SOD; 1:2000, Abcam, Cambridge, MA, USA), NAD(P)H dehydrogenasequinone

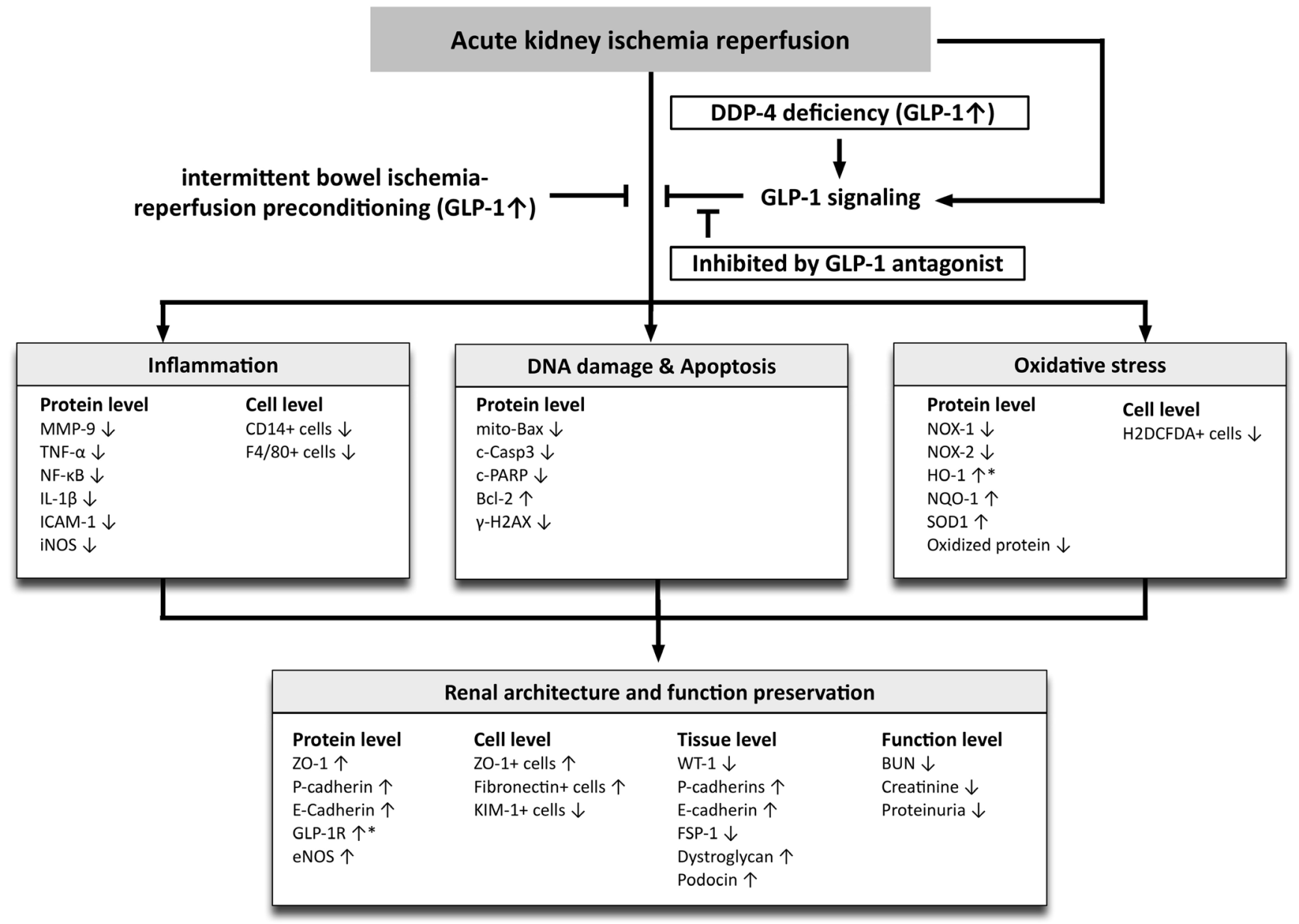

Figure 12: Proposed mechanisms underlying the positive therapeutic effects of DPP4 deficiency and small bowel ischemia-reperfusion (IR) preconditioning against acute kidney IR injury. GLP-1 = glucagon like peptide 1; DDP4 = dipeptidyl peptidase 4 deficiency; MMP = matrix metalloproteinase; TNF = tumor necrosis factor; NF = nuclear factor; IL = interleukin; ICAM = intercellular adhesion molecule; iNOS $=$ inducible nitric oxide synthase; mito $=$ mitochondrial; $\mathrm{c}$-Casp $=$ cleaved caspase 3; c-PARP = cleaved protein expression of Poly (ADP-ribose) polymerase; $\mathrm{HO}=$ heme oxygenase; $\mathrm{NQO} 1=\mathrm{NAD}(\mathrm{P}) \mathrm{H}$ quinone dehydrogenase 1 ; eNOS = endothelial nitric oxide synthase; KIM = kidney injury molecule; WT-1 = Wilm's tumor suppressor gene 1; FSP-1 = fibroblast specific protein $1 ; \mathrm{BUN}=$ blood urea nitrogen . 
1 (NQO 1; 1:1000, Abcam, Cambridge, MA, USA), NOX-1 (1:1500, Sigma, St. Louis, Mo, USA), NOX-2 (1:750, Sigma, St. Louis, Mo, USA), and actin (1: 10000, Chemicon, Billerica, MA, USA). Horseradish peroxidaseconjugated anti-rabbit immunoglobulin IgG (1:2000, Cell Signaling, Danvers, MA, USA) was used as a secondary antibody for $1 \mathrm{~h}$ at room temperature. Then, after washing, the blots were developed and visualized by enhanced chemiluminescence (ECL; Amersham Biosciences, Amersham, UK) and exposed to Biomax L film (Kodak, Rochester, NY, USA). ECL signals were quantitated using Labwork software (UVP, Waltham, MA, USA).

\section{Oxyblot oxidized protein detection}

Oxidative stress was assessed according to previously published protocols [13-15] using the Oxyblot Oxidized Protein Detection Kit (S7150, Chemicon, Billerica, MA). First, we DNPH derivatized $6 \mu \mathrm{g}$ total rat kidney protein for 15 minutes according to the manufacturer's instructions. Then, the DNPH derivatized proteins were electrophoresed on a $12 \%$ SDS-PAGE followed by electrophoretic transfer of proteins to nitrocellulose membranes. After blocking, the membranes were incubated with primary anti-DNP1 antibody (1:150) for $2 \mathrm{~h}$ followed by incubation with secondary antibody (1:300) for $1 \mathrm{~h}$ at room temperature. After washing repeatedly, the blots were developed and visualized by enhanced chemiluminescence (ECL; Amersham Biosciences, Amersham, UK) and exposed to Biomax L film (Kodak, Rochester, NY, USA). ECL signals were quantified using Labwork software (UVP, Waltham, MA, USA). A standard control was loaded on each gel as reference for oxyblot protein analysis.

\section{Assessment of ROS by H2DCFDA}

We assessed reactive oxygen species (ROS) according to previously published protocols [34]. Thirty minutes prior to the end of the study, the rats were anesthetized with $2 \%$ isoflurane and intravenously administered $150 \mu \mathrm{g}$ of CM-H2DCFDA (Life Technologies Molecular Probes; $100 \mu \mathrm{g}$ of H2DCFDA was dissolved in $200 \mu 1$ PBS). After 30 minutes, the kidneys were harvested and immediately frozen in liquid nitrogen. Then, $5 \mu \mathrm{m}$ thick cryostat sections were cut at $-20^{\circ} \mathrm{C}$ and the serial frozen sections were fixed with $4 \%$ paraformaldehyde in phosphate buffered saline at $4{ }^{\circ} \mathrm{C}$ for 5 minutes. After washing the sections with PBS, they were co-stained with DAPI and analyzed by fluorescence microscopy.

All sections were examined under a fluorescent microscope $(200 \times)$. The fluorescence and gray photos were assessed by DP controller 2.1.1.183 (Olympus). The gray photos were used to measure fluorescence intensity with Image J 1.37v (National Institutes of Health, USA). Three gray photos from three sections were randomly analyzed for each animal. The baseline fluorescence intensity (BFI) [arbitrary unit/200 $\times$ high-power field (HPF)] was determined by comparing the area of increased fluorescence intensity (IFI) with the area in the kidney without $\mathrm{H}_{2}$ DCFDA. Six BFI areas were measured from each gray photo. The mean IFI and BFI were calculated from three randomly chosen BFI areas. The relative fluorescence intensity was determined from the ratio of IFI to BFI.

\section{Statistical analysis}

Quantitative data was expressed as mean \pm SD. Statistical analysis was performed by ANOVA followed by Bonferroni multiple-comparison post hoc test with the SAS statistical software for Windows version 8.2 (SAS institute, Cary, NC). A $P<0.05$ was considered statistically significant.

\section{Authors' contributions}

YTC, CGW, and HKY conceived the study, and participated in the design, data acquisition and analysis as well as drafting the manuscript. $\mathrm{CCY}, \mathrm{CHC}, \mathrm{KHC}$, PHS, YLC, and HTC conducted the laboratory assays and troubleshooting. SYC, SC, FYL, SFK, and MSL performed data acquisition, analysis, and interpretation. All authors read and approved the final manuscript.

\section{ACKNOWLEDGMENTS}

This study was supported by a program grant from Chang Gung Memorial Hospital, Chang Gung University (Grant number: CMRPG8E0221).

\section{CONFLICTS OF INTEREST}

The authors declare that they have no conflicts of interest.

\section{REFERENCES}

1. Hoste EA, De Corte W. Implementing the Kidney Disease: Improving Global Outcomes/acute kidney injury guidelines in ICU patients. Curr Opin Crit Care. 2013; 19:544-553.

2. Johnson RJ, Feehally J, Floege J. Comprehensive clinical nephrology, 5th edition. Philadelphia, Penn: Elsevier/ Saunders. 2014; pp 1320.

3. Kumar A, Singh NP. Antimicrobial dosing in critically ill patients with sepsis-induced acute kidney injury. Indian J Crit Care Med. 2015; 19:99-108.

4. Mehta RL, Kellum JA, Shah SV, Molitoris BA, Ronco C, Warnock DG, Levin A, Acute Kidney Injury Network. Acute Kidney Injury Network: report of an initiative to 
improve outcomes in acute kidney injury. Crit Care. 2007; 11:R31.

5. Parikh CR, Coca SG, Wang Y, Masoudi FA, Krumholz HM. Long-term prognosis of acute kidney injury after acute myocardial infarction. Arch Intern Med. 2008; 168:987-995.

6. Reichel RR. Acute kidney injury: quoi de neuf? Ochsner J. 2014; 14:359-368.

7. Friedericksen DV, Van der Merwe L, Hattingh TL, Nel DG, Moosa MR. Acute renal failure in the medical ICU still predictive of high mortality. S Afr Med J. 2009; 99:873-875.

8. Sementilli A, Franco M. Renal acute cellular rejection: correlation between the immunophenotype and cytokine expression of the inflammatory cells in acute glomerulitis, arterial intimitis, and tubulointerstitial nephritis. Transplant Proc. 2010; 42:1671-1676.

9. Thadhani R, Pascual M, Bonventre JV. Acute renal failure. N Engl J Med. 1996; 334:1448-1460.

10. Zuk A, Bonventre JV, Brown D, Matlin KS. Polarity, integrin, and extracellular matrix dynamics in the postischemic rat kidney. Am J Physiol. 1998; 275:C711-731.

11. da Silva LB, Palma PV, Cury PM, Bueno V. Evaluation of stem cell administration in a model of kidney ischemiareperfusion injury. Int Immunopharmacol. 2007; 7:1609-1616.

12. Li B, Cohen A, Hudson TE, Motlagh D, Amrani DL, Duffield JS. Mobilized human hematopoietic stem/ progenitor cells promote kidney repair after ischemia/ reperfusion injury. Circulation. 2010; 121:2211-2220.

13. Chen YT, Sun CK, Lin YC, Chang LT, Chen YL, Tsai TH, Chung SY, Chua S, Kao YH, Yen CH, Shao PL, Chang KC, Leu $\mathrm{S}$, et al. Adipose-derived mesenchymal stem cell protects kidneys against ischemia-reperfusion injury through suppressing oxidative stress and inflammatory reaction. J Transl Med. 2011; 9:51.

14. Chen YT, Tsai TH, Yang CC, Sun CK, Chang LT, Chen HH, Chang CL, Sung PH, Zhen YY, Leu S, Chang HW, Chen YL, Yip HK. Exendin-4 and sitagliptin protect kidney from ischemia-reperfusion injury through suppressing oxidative stress and inflammatory reaction. J Transl Med. 2013; 11:270.

15. Yip HK, Yang CC, Chen KH, Huang TH, Chen YL, Zhen YY, Sung PH, Chiang HJ, Sheu JJ, Chang CL, Chen CH, Chang HW, Chen YT. Combined melatonin and exendin-4 therapy preserves renal ultrastructural integrity after ischemia-reperfusion injury in the male rat. J Pineal Res. 2015; 59:434-447.

16. Oeseburg $H$, de Boer RA, Buikema H, van der Harst P, van Gilst WH, Sillje HH. Glucagon-like peptide 1 prevents reactive oxygen species-induced endothelial cell senescence through the activation of protein kinase A. Arterioscler Thromb Vasc Biol. 2010; 30:1407-1414.

17. Shimoda M, Kanda Y, Hamamoto S, Tawaramoto K, Hashiramoto M, Matsuki M, Kaku K. The human glucagonlike peptide-1 analogue liraglutide preserves pancreatic beta cells via regulation of cell kinetics and suppression of oxidative and endoplasmic reticulum stress in a mouse model of diabetes. Diabetologia. 2011; 54:1098-1108.

18. Holst JJ, Deacon CF. Glucagon-like peptide-1 mediates the therapeutic actions of DPP-IV inhibitors. Diabetologia. 2005; 48:612-615.

19. Goke R, Larsen PJ, Mikkelsen JD, Sheikh SP. Distribution of GLP-1 binding sites in the rat brain: evidence that exendin-4 is a ligand of brain GLP-1 binding sites. Eur J Neurosci. 1995; 7:2294-2300.

20. Kinzig KP, D’Alessio DA, Seeley RJ. The diverse roles of specific GLP-1 receptors in the control of food intake and the response to visceral illness. J Neurosci. 2002; 22:10470-10476.

21. Tsai TH, Sun CK, Su CH, Sung PH, Chua S, Zhen YY, Leu S, Chang HW, Yang JL, Yip HK. Sitagliptin attenuated brain damage and cognitive impairment in mice with chronic cerebral hypo-perfusion through suppressing oxidative stress and inflammatory reaction. J Hypertens. 2015; 33:1001-1013.

22. Zerilli T, Pyon EY. Sitagliptin phosphate: a DPP-4 inhibitor for the treatment of type 2 diabetes mellitus. Clin Ther. 2007; 29:2614-2634.

23. Scheen AJ. Dipeptidylpeptidase-4 inhibitors (gliptins): focus on drug-drug interactions. Clin Pharmacokinet. 2010; 49:573-588.

24. Sun CK, Leu S, Sheu JJ, Tsai TH, Sung HC, Chen YL, Chung SY, Ko SF, Chang HW, Yip HK. Paradoxical impairment of angiogenesis, endothelial function and circulating number of endothelial progenitor cells in DPP4deficient rat after critical limb ischemia. Stem Cell Res Ther. 2013; 4:31.

25. Matsubara J, Sugiyama S, Sugamura K, Nakamura T, Fujiwara Y, Akiyama E, Kurokawa H, Nozaki T, Ohba K, Konishi M, Maeda H, Izumiya Y, Kaikita K, et al. A dipeptidyl peptidase-4 inhibitor, des-fluoro-sitagliptin, improves endothelial function and reduces atherosclerotic lesion formation in apolipoprotein E-deficient mice. J Am Coll Cardiol. 2012; 59:265-276.

26. Evrengul H, Celek T, Tanriverdi H, Kaftan A, Dursunoglu D, Kilic M. The effect of preinfarction angina on clinical reperfusion time in patients with acute myocardial infarction receiving successful thrombolytic therapy. Can J Cardiol. 2005; 21:915-920.

27. Yang X, Cohen MV, Downey JM. Mechanism of cardioprotection by early ischemic preconditioning. Cardiovasc Drugs Ther. 2010; 24:225-234.

28. Gent S, Skyschally A, Kleinbongard P, Heusch G. Ischemic preconditioning in pigs: a causal role for signal transducer and activator of transcription 3. Am J Physiol Heart Circ Physiol. 2016; 312:H478-H484.

29. Kerendi F, Kin H, Halkos ME, Jiang R, Zatta AJ, Zhao ZQ, Guyton RA, Vinten-Johansen J. Remote postconditioning. Brief renal ischemia and reperfusion applied before 
coronary artery reperfusion reduces myocardial infarct size via endogenous activation of adenosine receptors. Basic Res Cardiol. 2005; 100:404-412.

30. Heusch G. Molecular basis of cardioprotection: signal transduction in ischemic pre-, post-, and remote conditioning. Circ Res. 2015; 116:674-699.

31. Kanoria S, Robertson FP, Mehta NN, Fusai G, Sharma D, Davidson BR. Effect of Remote Ischaemic Preconditioning on Liver Injury in Patients Undergoing Major Hepatectomy for Colorectal Liver Metastasis: A Pilot Randomised Controlled Feasibility Trial. World J Surg. 2016; 41:1322-1330.

32. Bashir SO, Mostafa OA, Rizk MS, Al-Ridi MR, Morsy MD. Intestinal ischemic preconditioning modulates oxidative stress in rat's spinal cord ischemic reperfusion injury. Am J Biomed Sci. 2012; 4:220-232.

33. Stringa P, Lausada N, Romanin D, Portiansky E, Zanuzzi C, Machuca M, Gondolesi G, Rumbo M. Pretreatment Combination Reduces Remote Organ Damage Secondary to Intestinal Reperfusion Injury in Mice: Follow-up Study. Transplant Proc. 2016; 48:210-216.
34. Chen YL, Chen KH, Yin TC, Huang TH, Yuen CM, Chung SY, Sung PH, Tong MS, Chen CH, Chang HW, Lin KC, Ko SF, Yip HK. Extracorporeal shock wave therapy effectively prevented diabetic neuropathy. Am J Transl Res. 2015; 7:2543-2560.

35. Lin KC, Yip HK, Shao PL, Wu SC, Chen KH, Chen YT, Yang CC, Sun CK, Kao GS, Chen SY, Chai HT, Chang CL, Chen $\mathrm{CH}$, et al. Combination of adipose-derived mesenchymal stem cells (ADMSC) and ADMSC-derived exosomes for protecting kidney from acute ischemiareperfusion injury. Int J Cardiol. 2016; 216:173-185.

36. Chang CL, Sung PH, Sun CK, Chen $\mathrm{CH}$, Chiang HJ, Huang TH, Chen YL, Zhen YY, Chai HT, Chung SY, Tong MS, Chang HW, Chen HH, et al. Protective effect of melatonin-supported adipose-derived mesenchymal stem cells against small bowel ischemia-reperfusion injury in rat. J Pineal Res. 2015; 59:206-220. 